\title{
Self-Organization and Coherency in Biology and Medicine*
}

\author{
Alexander 0. Goushcha1\#, Tatiana 0. Hushcha², Leonid N. Christophorov³, \\ Michael Goldsby 4 \\ ${ }^{1}$ Institute of Physics, NAS Ukraine, Kyiv, Ukraine \\ ${ }^{2}$ Institute of Bioorganic Chemistry and Petrochemistry, NAS Ukraine, Kyiv, Ukraine \\ ${ }^{3}$ Bogolyubov Institute for Theoretical Physics, NAS Ukraine, Kyiv, Ukraine \\ ${ }^{4}$ University of California at San Diego, La Jolla, CA, USA \\ Email: "goushcha@cox.net
}

Received 18 July 2014; revised 18 August 2014; accepted 16 September 2014

Copyright (C) 2014 by authors and Scientific Research Publishing Inc.

This work is licensed under the Creative Commons Attribution International License (CC BY). http://creativecommons.org/licenses/by/4.0/

(c) (i) Open Access

\begin{abstract}
Self-organization has proven to be a universal functioning property inherent to the open systems, including biological entities and living organisms. The flux of energy or matter through the system enables its transition to a new ordered state, which results from a cooperative behavior of the system's constituents. The system functions far from thermodynamic equilibrium and its transitions between the states are treated within nonlinear models. An analysis of such behavior yields valuable information about the emergent properties of the particular system that is often impossible to obtain by other methods. This review summarizes some of the most interesting, recently reported phenomena related to dynamic self-organization and coherency at various complexity levels in living matter, demonstrating the widespread applications of these concepts in many modern fields of biological and healthcare research. The processes and interactions controlling self-organized behaviors are discussed in regards to molecular reactions, including mechanisms of protein folding, bioenergetics, and charge transfer. Phenomena in cells and tissues, as well as the examples of whole organs and organism levels are also reviewed. In addition, we analyze existing applications of self-organization and coherency processes in medicine. Special attention is given to determination of feedback mechanisms, control parameters, and order parameters needed to completely define the self-organized behavior and coherent dynamics of a particular system.
\end{abstract}

\section{Keywords}

Self-Organization, Nonlinear Dynamics, Order Parameter, Open Systems, Medicine, Systems Biology

"In memory of Valery Kharkyanen.

"Corresponding author. 


\section{Introduction}

\subsection{Self-Organization and Coherency as Inherent Properties of Biological Systems}

For many years, a mainstream approach in studies of complex biological entities was their dismantling with the purpose of examining individual components. Such an approach allowed incorporation of modern mathematical methods and theories in biological and healthcare research. Further progress was achieved from integrating the components of complex entities in interacting bottom-up and/or top-down systems. However, discovering a deductive link that explains emergent properties of a complex system from the properties of interacting components may not necessarily work, simply because such a deductive link may not exist [1]. Multiple studies elucidated a need to bring into play higher organizing principles available in nature and to apply them in experimental and theoretical studies, in order to find the answers to paradoxes emerging at all complexity levels — from biomolecules through cells to the most complex organisms [2]-[5]. The need to understand how the interaction of metabolites, proteins, genes, cells, or higher-level constituents can lead to unpredicted systems-level behaviors resulted in increased attention from the research community to the science of complex systems.

One of the main concepts in complex systems theory is self-organization. Self-organization is a process by which a system, consisting of interacting components, becomes ordered in space and/or time. This process means that the whole system has characteristics that differ qualitatively from those of the component parts without the interactions. The concept of self-organization, usually considered as a dynamic phenomenon in the systems far from thermodynamic equilibrium, was introduced by Prigogine [6] [7]. He discussed emergence of new properties and order-dissipative structures-in such systems, which would be stabilized due to energy exchange with an environment. To emphasize the role of cooperative (collective) effects in self-organization processes, Haken suggested naming this field of research synergetics [8] [9]. He suggested that the main problem of synergetics was finding the common principles that control the emergence of self-organizing structures. As consistent with synergetics, dynamically self-organizing systems rely on a continuous input of energy to be maintained (the so-called open systems) — which is exactly the case for functioning biological systems and living organisms. An important prerequisite for dynamic self-organization is the existence of a feedback between the components of the system. Such feedback initiates nonlinear dynamic properties and, if strong enough, complex behaviors of the components and the whole system, facilitating its transitions to new stable states.

The other important concept is coherency. In general, coherency may be considered as a property either in time or in space. Spatial coherence describes the correlation between entities or processes at different points in space. Temporal coherence describes the correlation or predictable relationship between entities or processes observed at different moments in time. Obviously, the coherency in biology borders closely with dynamic selforganization since it describes collective properties—synchronization-of entities or processes. In fact, coherence is a subset of dynamic self-organization phenomena since existence of a positive feedback between the components of a system is a prerequisite for self-organization to occur and is a precursor for coherency in the system.

To appropriately describe processes occurring in a complex, multi-dimensional system and to take into account the feedback, relevant mathematical models of a dynamically self-organizing system introduce the socalled order parameters, which in all cases are the generalized dynamic variables that reflect collective action of various sets of parameters of the system on its behavior [8]. For each specific system and within the adequate model, there could be at least one such generalized variable. Importantly, these order parameters may not necessarily represent directly measurable quantities and their values depend usually on a combination of the system's (microscopic) properties. The number of such influential properties is usually unknown, which emphasizes an imperative value of the order parameters. For any biological system, the motion of its components occurs on very wide time-scales (from femto seconds to hours), representing versatile dynamics. By introducing order parameters, nonlinear dynamics allows considering only those (slow) motions that determine the whole system dynamics on a microscopically long time-scale. Such slow modes integrate fast motions, which thereby could be excluded from consideration in the whole system model (slaving principle as defined by Haken [8] [10]). Thus, using order parameters as an important attribute of nonlinear dynamics allows developing low-dimensional representations of a high-dimensional system.

Selecting the order parameters for complex, multi-component systems might be rather challenging. In many cases, behavior of complex nonlinear dynamical systems can be analyzed using parameters that characterize divergence (uncertainties) of trajectories for the model's variables. The rate of such divergence (uncertainties) is 
described by characteristic variables of the relevant mathematical models-Lyapunov exponents and Kolmogorov (metric) or Shannon (information) entropy [8] [11]. Multiple studies showed that these variables correlate well with the order parameters' behaviors, uniquely characterizing various functional states (stable states, oscillations, spiral waves, deterministic chaos, and other attractors) of the system and allowing to use Lyapunov exponents and entropies in lieu of order parameters to analyze and predict emergent properties of the complex systems [12]-[15].

Applying concepts of dynamic self-organization allows not merely constructing an appropriate mathematical model for a complex biological system, but in many cases provides a clear path to understand the principles that drive the system's functions. There might be many important exogenous and endogenous interactions, feedback loops, etc. and it might be impossible to define or even to predict all of them immediately. However, introduction of the order parameters allows description of the whole system in its emergent properties, providing the way for validating experimental results. As soon as the model consistent with the experiment is built, further development may be focused on determination of important geometrical, physical, chemical, and physiological variables and properties of the system that stand behind the order parameters.

\subsection{Historical Aspects}

The concepts of self-organization and coherency have a long history in biology, dating back to the famous essay by Schrödinger [16]. He has pointed out that a remarkable biological order, functional stability, and energy efficiency could not be satisfactorily explained exclusively by statistical laws; supplementary principles of a collective origin should be applied.

Later, Fröhlich recognized a long-range biological order as coherent molecular excitations [17]. He admitted that coherent excitations and other coherency effects could be responsible for the mentioned remarkable properties of biological systems and considered crucial role of coherency (facilitated by feedback loops) for organism, suggesting a link of insufficiency or impairment of coherent dynamics, to cancer [18]. In its simplest form, the coherent excitation was suggested to occur in the form of a "giant dipole" vibration-a synchronous vibration of multiple dipoles in a biomolecule, which is stabilized in a non-equilibrium manner through the elastic deformability of the macromolecules. Within this approach, the electrochemical energy was considered to funnel into collective degrees of freedom in order to avoid thermal dissipation and enable biochemical work.

Soon after Fröhlich's works on coherent molecular excitations, the theory of biological coherence was further elaborated by Davydov, who described the transport of metabolic energy along biological polymers over distances, introducing the concept of the soliton [19]. The idea behind the Davydov's soliton is that the hydrolysis of adenosine triphosphate (ATP) creates excited vibrational states in the peptide group, allowing vibrational excitation to propagate to the neighboring groups due to dipole interaction between groups. The excitation interacts also with the hydrogen bond, creating a local deformation that is coupled (self-trapped) to the vibrational excitation through a feedback mechanism. If the feedback is strong enough, a new, non-dissipative state associated with vibrational excitation and hydrogen bond distortion will be created and will propagate coherently along the peptide chain. This concept proved to be productive for explaining the bioenergetics mechanisms at physiological conditions; it was further elaborated theoretically [20]-[23] and obtained some experimental evidence in support [23] [24]. Although additional experiments are required to test the role of excited vibrational states in protein function and bioenergetics in a more direct way, the Davydov's soliton concept as well as Fröhlich's "giant dipole" played an eminent role in triggering comprehensive studies of dynamic self-organization effects through coupling of localized excitations and protein conformational dynamics in biological systems.

Apparently, the first mathematical description of self-organization process in biological systems was the reaction-diffusion theory of morphogenesis by Turing [25]. Turing proved that morphogens in the cells could form concentration patterns through diffusion and chemical reactions under certain favorable conditions facilitated by catalysts or auto-catalytically. Turing's model relied on the principles later named "self-organization", in which the feedback between the components of a system plays an essential role. In his model, Turing assumed that the system was initially pushed out of equilibrium. Applying a linear approach he, however, considered only small fluctuations in the system. Still he noticed that in the event of large-magnitude fluctuations, the system should behave in a similar way since other (external) parameters would limit its behavior. Turing indicated that only a few 'leading' terms in his equations dominated the system's behavior after a "lapse of time”. The leading terms determined concentration variables that produced periodic patterns on a long time scale, which suggested an 
imperative role of appropriately selected variables as the order parameters. In support of Turing's ideas, morphogenesis was described later using self-organization models by Kauffman [26]. Similar models were subsequently explored by many scientists discussing emergent effects within the modern dynamic self-organization paradigm of a system with strong feedback loops on different time scales, using chemical concentrations as the order parameters [27] [28].

To date, dynamic self-organization effects were reported at different complexity levels in biological systems - in molecules, cells, tissues, organs, and organ systems. It is widely understood that synergetic, chaotic, and coherent behaviors stimulated by feedback dominate physiological systems. This was evidenced by experimental studies and had also been supported by very successful modeling [26] [29]-[39]. The concept of dynamic self-organization also received considerable attention by systems biology and healthcare scientists [40]-[42]. The number of research papers on self-organization effects in biology and living organisms has mounted exponentially during the last decades. This review summarizes those of the most recent studies that relied on dynamic self-organization concepts in the sense of Prigogine [6] [7] and Haken [8] [9], pinpointing whenever was possible the order parameters, control parameters, feedback mechanisms, and emphasizing importance of slaving principle in the systems' behavior.

\subsection{Outline of This Review}

The remainder of this article is organized as follows. Section 2 summarizes the most important recent works on dynamic self-organization in various biological systems and briefly analyzes suggested order parameters and control parameters. The section covers processes occurring in protein folding, motor proteins, charge transfer, and receptor biomolecules. Significant attention is paid to cellular level systems with the emphasis on cytoskeleton, cardiac myocytes, and neurons. The description of self-organization studies in organs and organism level systems concludes this section. Section 3 introduces existing applications of self-organization and coherency concepts in medicine and medical research. It is shown that health disorders and diseases can be described in terms of coherency and self-organization loss. An important role of models based on bottom-up and top-down approaches is discussed. Section 4 analyzes ways of defining the order parameters at different complexity levels of self-organizing biological systems and in medicine. Section 5 concludes this review and outlines promising areas for future studies.

\section{Biological Systems}

\subsection{Macromolecules}

a) Molecular paradoxes

Historically, research on dynamic self-organization in macromolecules has been increasingly focused on the mechanisms of protein folding, dynamics of ion channels of biomembranes, and non-thermal (i.e. coherent) modes of energy transfer. Conventional theories did not explain how the folding protein chooses a single stable structure among billions of others (the so-called Levinthal paradox [43]), how the freely movable ion channels organize into metastable periodic patterns which finally evolve into clusters [44], the mechanisms of non-thermal biological effects of weak electromagnetic fields with a quantum energy far below the average energy of thermal fluctuations (see e.g. [45]), etc. These and many other paradoxes at the biomolecular level were subsequently explained, taking into account the importance of dynamic self-organization and coherency effects in biological systems and living organisms.

b) Protein folding

It is now widely accepted that protein folding occurs essentially through dynamic self-organization. The most successful models consider the protein folding as a two-state first-order phase transition [46] triggered by a spontaneous nucleation [47] and progressing along the energy funnel-type landscape [48] by choosing thermodynamically most favorable barrier-crossing steps, facilitated by favorable conformational changes [49] and collective dominance of native-like interactions over alternative combinations of interactions [50]. The funnel landscape in the proposed models emerges only for those amino acid sequences for which there is a structure in which nearly all the various interactions are simultaneously minimized; i.e., native proteins are only minimally frustrated [50]. Using the language of dynamic self-organization, such processes may be formalized in terms of non-equilibrium phase transition of the disorder-order type with the frustration parameter as one of the important 
order parameters.

The manner in which a protein folds into its native conformation was also examined in terms of a structural self-organization process applying topological methods [51], in which a specific topological quantity that characterizes the folding pathways was suggested as the order parameter.

Recent progress in understanding the most fundamental aspects of polypeptide self-organization into native conformations facilitated further research into regulatory mechanisms that underpin their ability to adapt to changing conditions [52]. The self-organization of the protein folding process augments itself by enhancing the stability of the core against large-scale motions that would unfold the protein [53], stabilizing the native state through hydrophobic interaction that drives the overall collapse of the chain [54], robustness of the native structure against mutations [55], etc. Available experimental results support modern understanding of physical principles of dynamic self-organization in protein structures, emphasizing such important features of this process as acquiring the unique protein structure among alternatives, nucleation of the folding process and meta stable folding intermediates, cooperation among neighboring domains in a folding protein, as well as folding and selforganization effects in various disease states [56]-[61].

A number of works indicated, though, that a unifying concept that combines the basic features governing self-organization of proteins into complex three-dimensional structures in vitro and in vivo is still lacking [62] [63]. This may be attributed to a complexity of the folding process that involves a very large number of interactions (with structural feedback), allowing calculation of only relatively small native structures and applying other critical limitations. Current studies are focused on numerous as yet unresolved questions, such as unfolding-refolding reactions and hidden intermediates [58], role of intramolecular interactions in folding [64], effects of water dynamics in slaving the chain movements [50], and other topics.

c) Motor proteins

Motor proteins are molecular motors powered by the hydrolysis of ATP, converting chemical energy into mechanical work. Examples of molecular motors include cytoskeletal motors (myosins, kinesins, dineins), polymerization motors (actins, microtubules, dynamins), rotary motors, nucleic acid motors, and others. Pattern formation through self-organization and oscillatory behavior of motor proteins has been known for many years (see e.g. [65]-[67]). Effects related to the feedback in the protein-environment system and their role in the function of motor proteins were evaluated recently in a number of studies.

The level-arm versus Brownian motor models of motor proteins were discussed, demonstrating that either the time scale difference in conformational changes or switching of asymmetric potential (both driven by ATP) may facilitate the necessary feedback to control the operation of motor proteins [68]. The function of molecular motors by transitions between different conformations facilitated by a sustained influx and outflow of energy was rationalized in terms of non-equilibrium thermodynamics of an open system with feedback [69], in which free energy and entropy functions could be considered as the order parameters.

A nonlinear dynamic model for an ensemble of motors was proposed to describe their transition from a stable to unstable operating mode, suggesting a load force as the order parameter and several potential control parameters influencing the feedback: biochemical signaling that modulate the load force, mechanical signaling including the rate of load-accelerated dissociation of motors from the filament, and the number of active motors that can act collectively [66] [70]. The cooperation of a large number of such motors can lead to instabilities and transitions to different operational modes (attractors) of the system, revealing hysteretic behavior, bifurcations, and spontaneous oscillations. For example, self-organized dynamic redistribution of dynein motors was suggested to account for the nuclear oscillations in the motor-microtubule system. Dynein motors, responding to load forces, cooperatively detach from the trailing microtubules. After redistributing via the cytoplasm, they attach along the leading microtubules, thereby generating the force asymmetry necessary for the oscillations [71]. These cooperative effects were formalized using the equation for microtubules dynamics with forces exerted by the attached motors. Suggesting the concentration of attached motors as the order parameter with load forces as control parameters revealed attractors and oscillating solutions typical for dynamically self-organized systems.

d) Ion channels of biomembranes

Systematic studies of ion channels in biomembranes started with the seminal work by Hodgkin and Huxley [72], in which they discussed excitable membranes and presented a first model describing experimentally observed kinetics of ion transfer through the channel and its correlation with the channel gating. It was also shown that the open probability of a sodium channel increases as the membrane's depolarization degree is increased, facilitating the positive feedback such that channel opening allows more positively charged ions to enter the cell, 
shifting the membrane potential towards depolarization and in turn opening more channels [73]. An intrinsic tendency for self-organization in ion channels with electro-diffusion was first analyzed over 25 years ago [44]. It was shown that when a concentration gradient of salt across the membrane exceeds a certain threshold, freely movable ion channels may organize into transient periodic patterns, which is typical for the systems driven away from a thermal equilibrium. Such correlated behavior of structural elements of the channels was discussed later in many works [74] [75].

Generally, the ion transport through the channel is considered using the idea of a flexible structure of the channel-forming proteins that channel ions; they carry currents that change the cell voltage, which in turn changes the ion channel properties [76]. A novel approach suggesting existence of a strong positive feedback due to interaction of the transferred ions with a flexible structure of the channel forming proteins, facilitating formation of the states with different channel conductivities through dynamic self-organization mechanism was discussed in the early nineties [77]. Among the features enhancing the feedback mechanisms, a strong ion-conformational interaction and markedly different time scales for the events of ion transfer through the pore and conformational relaxation of pore-forming proteins were indicated (emphasizing importance of the slaving principle). As the order parameter driving formation of different conductivity states, the authors considered generalized structural variables defined by free energies of the components of a functioning ion channel.

Recent studies on high-resolution x-ray structures, molecular dynamics (MD) simulations, NMR spectroscopy, and computer modeling showed distinctly different structures for the gated ion channels in the open-pore (active) and closed-pore (resting) conformations [78]-[84]. It was also reported that the mean time for the passage of a single ion through a channel is several orders of magnitude shorter than conformational changes associated with channel gating events [79] [84]. Such a pronounced difference in time scales should be considered as a prerequisite for a strong feedback and emergent nonlinear dynamical properties in a system as discussed in [77] [85]. However, currently available x-ray structures of the channels in various conformations, as well as time limitations for extended MD calculations, do not allow correlating accurately conformational changes within the channel forming proteins with the ion transport events. Additional studies are required to clarify whether these conformational differences have functional relevance and to understand the magnitude of possible feedback in the system of transported ions and channel-forming proteins.

A number of studies explored cooperative interactions between ion channels using bifurcation analysis and stochastic simulations models [86], proving that cooperativity and anti-cooperativity can modify the range of sustained firing and cell-intrinsic noise, induce multi stability, and account for other experimental observations.

The cooperative behaviors of ion channels were discussed in many other works, showing that the positive feedback facilitates synchronous opening of all channels in the ensemble [73] [87]. Calcium oscillations, which exemplify one of the most important signaling mechanisms in cell biology, were also discussed within the concept of collective behavior of ion channels. According to recent studies, a coherent wave of calcium release, triggered by stochastic release events from a group of intracellular ion channel clusters, sweeps the cell. Oscillations in this model were believed to emerge through a spatial coherence resonance mechanism [88], i.e. a disorder-order phase transition.

e) Photosynthetic systems

One of the functions of the photosynthetic systems is charge separation across the biological membrane triggered by a light-absorption event. This function is performed by specific cofactor-protein complexes-reaction centers (RCs) - embedded in the photosynthetic membranes. Numerous studies discuss coupling between the photo-induced charge transfer and conformational dynamics in RCs [89]-[92]. First discussions of dynamic selforganization in functioning RCs considered the possibility for such coupling to cause conformational changes that relax on a much longer time scale than the electron transfer/recombination events, causing a kind of structural memory in the cofactor-protein environment [93] [94] and allowing applying the slaving principle in modeling the channel's function. Recent experimental studies of photosynthetic RCs proved existence of the chargeseparation induced, long-lived conformational changes that relax orders of magnitude slower than the charge transfer events [95]-[97], which in accord with the previously developed theories should create a strong feedback between transported charges and protein-cofactor environment of RCs, initiating dynamic self-organization behavior [93] [94].

Experimental results available to date were formalized theoretically within the concept of dynamic self-organization in the ensemble of RCs, specifying charge-transfer induced conformational changes and related free-energy functions as the order parameters [94] [98]. Some of the experimental findings reported in those 
works did not allow explanation outside the nonlinear dynamics theories. The same concepts were applied recently to develop a single-molecule model of photo induced charge transport in RCs, also suggesting its experimental verification [99].

The role of dynamic self-organization in the photosynthetic electron transfer was additionally discussed within a general theory of phase transitions in far from thermodynamic equilibrium biological systems with active friction [100]. In separate studies, self-organization effects were reported in light-harvesting complexes of photosynthetic membranes in terms of supra molecular protein arrangement and function in grana thylakoids [101].

Recent experiments with light harvesting complexes of photosynthetic systems have provided conclusive evidence that relatively long-lived quantum coherent states exist in the electronic and vibrational sub-systems of biological molecules [102]-[104]. Remarkably, the long-lived coherent effects in photosynthetic systems were also reported at room temperature [105] [106]. These experiments demonstrated that light-induced excitation energy is collectively shared among the light-harvesting molecules, allowing excitation to move coherently in space and enabling efficient energy harvesting and trapping.

f) Rhodopsins, receptor and signaling proteins

G-proteins (guanine nucleotide-binding proteins) are a family of membrane signaling receptors involved in processing signals received by cells and transmitting signals between the cells. They communicate signals from many photoreceptors, hormones, neurotransmitters, and other signaling factors. Studies of optically coupled neuronal function of these receptors revealed bistable behaviors with a pronounced feedback of synaptically released glutamate on neurotransmitter release [107].

Rhodopsin is a G-protein-coupled receptor of the retina that is responsible for the initial events of light sensing. Dynamic self-organization effects were shown to be important in rhodopsin expression. Explaining this suggestion, it was noted that positive feedback loops in photoreceptor cells create bistability in the network of activators and repressors, thereby controlling the cell-type specific Rhodopsin expression in a self-organized manner [108].

Rhodopsin is extremely sensitive to light; it mediates transduction of single photons into transient electrical responses. The role of calcium ions and other factors in regulation of rhodopsin's response to light was studied in many works (see e.g. [109] [110]). The most recent data showed that high fidelity of rhodopsin's photoresponse is a collective function of biochemical, physical, and geometrical components, also emphasizing that calcium controls negative feedback loop, suppressing output electrical signal at high incident light intensity ("eye adaptation”) [111] [112]. The electrical transient generation involved stochastic nonlinear dynamics of the signaling cascade (avalanche) with a pronounced positive feedback loop, which ensured high electrical response to low-intensity incident light [112]. In the sited work, the number of active molecules was conventionally used for the order parameter with the calcium concentration as one of the control parameters.

In biomolecular signal transduction, dynamic self-organization effects in switching of protein signal transduction cascades to bistable and/or multi-stable operation modes was formalized using the normalized populations of phosphorylation sites as the order parameter, with the particle number and catalytic enzyme concentrations serving as control parameters [113].

The research on coherent effects in photoreceptor proteins extended to retinal isomerization in bacteriorhodopsin [114] and coherent vibrational dynamics in rhodopsins [115] [116].

Among the other macromolecules exhibiting coherent behaviors, we would like to mention bacterial cytochrome c oxidase, in which the protein moiety and cofactor motions leading to altered conformations can be coherent rather than stochastic in nature. Such coherent motions was suggested to play a key role in controlling the photo-initiated transfer of carbon monoxide between binding sites and explain the high efficiency of the reaction [117]. The authors suggested a generalized reaction coordinate as the order parameter that involved conformational relaxation of the haem group with ligand transfer contribution.

\subsection{Cell and Tissues}

a) Cytoskeleton

One of the central questions in modern cell biology is how large macroscopic cellular structures are formed and maintained. It is unknown what determines the various shapes and sizes of cellular organelles, why specific structures form in particular places, and how cellular architecture is affected by function and vice versa. Recent discovery demonstrated how the size of the cells could be controlled: motor proteins walk along the microtu- 
bules, reaching the end, at which point they collectively depolymerize longer microtubules faster than shorter ones, providing feedback necessary to control the length [118].

The cytoskeleton, an organized network of filamentous proteins, is an essential component of all eukaryotic cells. It plays a major role in morphogenesis, transport, motility, and cell division. An important cytoskeletal structure in various cell types is the cortical rings formed by bundles of filaments that wrap around the cell. Such rings form within the cell cortex, a thin layer of filament network located close to the cell membrane. As a result of continuous consumption of fuel (ATP) and related active processes, dynamic patterns of filament orientation and density emerge via instabilities, leading to the formation of stationary and oscillating rings via self-organization and coherency phenomena [34] [119] [120]. Recent experimental and theoretical studies of spatiotemporal dynamics of actomyosin networks exemplified nonlinear dynamical properties of cytoskeleton [121], suggesting the global alignment of filament movement as the order parameter and using actin concentrations and myosin surface densities as control parameters.

The periodic changes in the cell's shape caused by interactions within a complex network of feedbacks between the components of the cell were known for over 25 years (see e.g. [122] [123]). Cells' contractility relies on a contractile complex of actin and myosin (actomyosin), in which myosin molecular motors convert chemical energy from ATP hydrolysis into forces on actin filaments. Most recent works demonstrated that periodic deformation of the shell shape could not be explained within the linear models of interaction between the components of the cell, but could be readily attributed to effects of nonlinear couplings between various dynamic modes of actomyosin networks, responsible for the cell motility. The motions of cells and organelles are highly coordinated via mechanical signaling, driven by motor proteins moving along cytoskeletal filaments. Moreover, actomyosin can self-organize and respond to mechanical stimuli through multiple types of biomechanical feedback [70] [124].

The cells' contractility was suggested to occur above a threshold concentration of myosin motors and at a critical distance between the bundles of motors and within a window of cross-link concentration [125]. The suggested mechanism of contraction was based on myosin filaments pulling neighboring bundles together into a cooperative, aggregated structure. The microscopic dynamic models of experimentally observed pulsatile behavior incorporated essential aspects of actomyosin self-organization: the asymmetric load response of individual filaments, the correlated motor-driven events of motor-induced filament sliding, and the complex competition of cross linking molecules and motor filaments in the network [126] [127].

The models developed thus far suggested various possibilities to define the order parameters that drive the system's dynamics - the number of motor proteins per cluster within a filament, free energy change for the constituents, polarization of polar filaments, and geometrical variables (e.g. a cortical layer thickness). These studies considered using the ATP, calcium, and myosin concentrations, connectivity and coupling in the network of filaments and motor proteins, as well as the asymmetry of a filament load response as control parameters that influence cytoskeleton nonlinear dynamics. A coupling between the activity of the cortical layer and calcium channels in the outer membrane, which are gated by the local stretching of the cortical layer, was also suggested as the control parameter. An increase of the extracellular calcium density enhances the actomyosin contractility in the cortical layer, providing the necessary feedback and facilitating sustained shape oscillations of the shell, (see e.g. [128]).

b) Cardiac myocytes

Cardiac myocytes' behavior is commonly considered in terms of dynamic self-organization formalism (see e.g. [129]-[131]). Spatiotemporal calcium dynamics within the cell has been demonstrated to occur as calcium sparks, short livedcalcium waves, full calcium waves, and spiral waves initiated by groups of calcium channels_calcium release units (CRUs) [132]-[135]. At the whole-cell level, these sub-cellular calcium dynamics give rise to the whole-cell calcium transients (and following beats) as a response to action potential, with the transient strongly dependent on the form of the sub-cellular calcium waves [5] [136].

Recent studies facilitated deeper insights into the mechanisms of calcium beat alternants in myocytes, demonstrating how disordered behaviors dominated by stochastic processes at the subcellular level become organized into beats alternating patterns at the whole cell level [131] [137]. Calcium release by an individual CRU was suggested to maintain the coherent pattern of release producing macroscopic alternations of calcium release, stable against stochastic de-phasing. The coherence within individual CRU and between CRU's was found to be facilitated by local coupling through calcium diffusion or globally by interactions through the membrane voltage. This result demonstrated that the emergence of calcium alternants at the whole cell level is a strongly coopera- 
tive phenomenon mediated by the diffusive coupling of a large number of CRUs. The transition from the "no alternants" to "sustained alternants" regime represents an onset of a new ordered pattern in calcium release by the whole cell through bifurcations, a fundamental topic of dynamic self-organization in open systems far from thermodynamic equilibrium.

Theoretical descriptions of self-organized behaviors on the sub-cellular level suggested the trans membrane potential and intracellular calcium concentration as order parameters in nonlinear dynamical models. On the cellular and tissue levels, the calcium alternants amplitude and number of synchronized sparks in a single beat were used as the order parameters with a variety of control parameters - the coupling efficiency between voltage and calcium concentration, coupling between neighboring CRUs, CRU recovering rate, cell-to-cell conduction, and others.

The pathological voltage oscillations, called early after-depolarizations, have been widely observed under disease conditions in cardiac cells. Recent studies proved their bifurcation origin using Lyapunov exponents analysis and other approaches [138]. During period pacing, chaos always occurs at the transition to early after-depolarizations as the stimulation frequency decreases, providing a distinct explanation for the irregular voltage oscillations observed in experiments.

Numerous works on spiral waves propagation in cardiac tissues discussed experimental observations using the concepts of nonlinear dynamics (see e.g. [139] [140]. These features of cardiac myocytes were suggested to affect functioning of a higher-level organization system—tissues and organs [4] [39] [141]. The cardiac myocytes as the components of an organ alter the behavior of the heart and the heart in turn alters the behavior of the components, yet both components and the heart are integrated in a higher multi-cellular structure, the organism. Apparently, such multi-level organization with feedback supports usage of dynamic self-organization ideas to describe the system function. However, it also calls for additional experimental and theoretical studies within the systems biology paradigm, also emphasizing the importance of relating specific order parameters to biophysical properties of calcium channels currents and pumps [5].

c) Neurons and neural networks

Early applications of nonlinear dynamics paradigm in neuroscience targeted mainly higher organization level entities-neural networks, tissues, and the whole brain, emphasizing the feedbacks between different complexity layers [142]-[145]. Accumulation of experimental data and further theoretical developments facilitated deeper understanding of self-organization properties of neurons, synapses, and neural networks, which we analyze briefly below.

Multiple experimental and theoretical works proved nonlinear dynamic behavior of single neurons [146]-[151]. In response to external stimuli, neurons generate electrical spikes and chemical signals, switching between different functional modes (attractors), showing bistability, multistability, oscillations, and chaotic behavior. Such switching between attractors is a typical dynamic self-organization process for a system far from thermodynamic equilibrium. Various mathematical models were applied to describe such emergent properties, allowing additional prediction of important functional details in neurons.

As an example, the models derived from Hodgkin-Huxsley approach [72] demonstrated the onset of mutistability, oscillations, bursting, and deterministic chaos using the gating variables describing the mean fraction of open gates of the sodium and potassium channels as the order parameters [147] [152]-[154]. The models used ion concentrations, transient input stimuli, coupling strength of the dendrite internal feedback connection, and the feedback time delay as control parameters that switch the neuron between attractors. Using Lyapunov exponents analysis of the chaotic neuron model, the internal state of the neuron-the generalized structural variable was suggested as the order parameter to model the onset of deterministic chaos, using the strength of the refractoriness and external stimuli as control parameters [155].

Complex functional properties have been proven for the components of a neuron, calling for application of nonlinear dynamics models on the lower organization level entities, see works on dynamics of dendritic spines [156], dendritic branch-specific plasticity [157], and vesicle transport dynamics [158].

Synapses are the macromolecular structures responsible for transmitting electrical or chemical signals between neurons. The first description by Hebb proposed a basic mechanism for synaptic plasticity, suggesting the imperative role of repeated interactions between neurons to accumulate and increase synaptic efficacy [159]. Such accumulation occurs through a versatile network of feedbacks acting on largely different time scales within a synapse, thereby suggesting the slaving principle and providing prerequisites for dynamic self-organization.

Synapses reveal complex dynamics that depend on the frequency and timing of presynaptic spike firing [160] 
[161], dendtritic spine dynamics [162], intracellular signaling [163], and other factors [164] [165]. Most recent biologically relevant models of synaptic plasticity emphasize their nonlinear dynamical behavior. One of the nonlinear models introduced the mechanism that adjusts the synaptic coupling to the neural activity through spike timing-dependent plasticity, allowing the synaptic strength to be either facilitated or depressed depending on the order of the spikes of pre- and post-synaptic neurons [166]-[168].

Synaptic strength was shown to depend on a crosstalk coming from neighboring synapses. The crosstalk may be due to various factors, e.g. dendritic diffusion of calcium or other intracellular diffusion processes, creating bifurcations in developmental synaptic plasticity and tending to destroy it. The related nonlinear dynamic models revealed switching to emergent functioning modes—oscillations or other attractors [169] [170]. Some studies showed, however, that the crosstalk might facilitate synaptic strength leading to self-organization of the synaptic connectivity. At an optimal crosstalk level, the amount of synaptic coupling gets maximal in a resonance-like manner preserving the existing level of collective dynamics in the brain by neutralizing the impact of random perturbations [171]. The corresponding nonlinear dynamic models used synaptic strength and synchronization parameter that characterized the phase difference of firing neurons as the order parameters, with the magnitude of a random synaptic input (crosstalk) as one of the control parameters.

Multiple studies indicated that a cooperative amplification of the synaptic efficiency by dentritic spines promotes nonlinear dendritic processing and associated forms of plasticity and storage, thus fundamentally enhancing the computational capabilities of neurons [172] [173]. The sign of synaptic plasticity was shown to be regulated by the action potential feedback to the synapse, thereby providing a mechanism for associative learning through a nonlinear dynamic mechanism [174].

Neural networks are multifunctional-they transduce sensory information, recover hidden signals, and generate new information. Researchers unanimously agree that neural networks function as self-organized systems far from the thermodynamic equilibrium. Below we will analyze only a few representative examples to demonstrate important features of biologically relevant nonlinear dynamic models.

Self-organization in neural networks relies on feedback processes that optimize biologic functions by correlating firing of groups of neurons to strengthen or modify synaptic connections between them, while the strengthened connections will in turn amplify the correlated firing of the neurons. Multiple experimental and theoretical studies explored nonlinear dynamic behaviors in groups and networks of neurons, confirming typical selforganized properties of their functioning like cell self-assembly, formation of standing patterns and other spatial structures, population bursts, consolidation of the synaptic changes, deterministic chaos, oscillating and propagating waves in synaptically-coupled networks, etc., as well as analyzing conditions of switching between different regimes of functioning (see e.g. [175]-[179] and references therein).

Successful models for excitatory and inhibitory neural networks were developed showing multi-stability onset with oscillations, spiral waves, and chaos. These models used the portion of cells receiving at least threshold excitation and average activity level of neuronal population as the order parameters [180]-[182]. The control parameters included the external stimulus intensity, proportion of firing inhibitory cell, synaptic firing rate, and number of synaptic connections.

Most of the models based on the chaotic neural networks used Lyapunov exponents and metric entropy analysis to explore the onset and properties of deterministic chaos and other dynamic regimes in neural networks, see for example [155].

The "self-organizing recurrent network" introduced recently combines several distinct forms of (nonlinear) synaptic plasticity to explore spatio-temporal patterns that mimic the properties of the biological systems [183] [184]. It was shown that all of the analyzed plasticity mechanisms were essential for the onset of emergent properties through self-organization. The model produced bursts, oscillations, steady-state operation dependently on connections and switching of synaptic activities. The fraction of active excitatory neurons was chosen as the order parameter, with the synaptic strength and extent of connections between cells as the control parameters.

The neuronal coherence was suggested to mechanistically control neuronal communication. Only coherently oscillating neuronal groups can interact effectively, because their communication windows for input and for output are open at the same times [185]. The so-called gamma-frequency oscillations in the brain tissues underlie various cognitive and motor functions. During gamma oscillations, individual neurons spike irregularly, but the collective dynamics of the local network are oscillatory. These oscillations exhibit pronounced coherence properties, but the exact mechanisms are still poorly understood [186]. 
d) Other examples at cell and tissue levels

Multiple studies confirmed that the dynamic properties of cellular structures are consistent with a role for selforganization in their formation, maintenance, and function; therefore, self-organization is considered to be a general principle in tissue organization and function [36] [38] [187] [188].

Meiotic nuclear oscillations crucial for proper chromosome pairing and recombination in yeast cells [71] as well as cellular biochemical processes responsible for e.g. self-regulating gene networks and phosphorylationdephosphorylation signaling [189] were also widely discussed within the concept of dynamic self-organization in far from thermodynamic equilibrium systems with feedback.

Successful nonlinear dynamical models have been developed in the studies of the cell cycle [190], bacterial chemotaxis [191], cell differentiation in response to growth factors [192], and other phenomena.

\subsection{Organs and Organ Systems}

a) Cardiovascular system

In cardiac physiology, the feedback between the participating components at several levels was proven to play an essential role in system functioning, suggesting inherent nonlinear dynamic properties [4] [39] [193] [194]. Numerous applications of nonlinear dynamics and stochastic methods to cardiac physiology have been discussed, emphasizing deterministic chaos [195], spiral waves as the precursors of chaotic behavior [139] [140], oscillatory patterns [196], and other nonlinear dynamic properties of the cardiovascular system [197]-[199]. Using Lyapunov exponents and entropy as order parameters proved to be a productive approach to pinpoint specifics of dynamic self-organization effects in cardiovascular systems.

One of the most promising efforts in modeling of the cardiac dynamics is associated with the study of the spatio-temporal evolution of the cardiac electrical activity. The early theories were based on extensions of the Hodgkin-Huxley nerve impulse equations [72]. In the current models, the cardiac tissue is considered to be an excitable medium of which the electrical and mechanical activity is based on the integration of control and feedback processes. Cardiac electrical activity controls cardiac mechanics via calcium concentration-membrane voltage coupling [136], whereas excitation-contraction interaction and changes in tissue length and/or tension affect electrophysiological properties via mechano-electrical feedback [200].

Using the heart ventricular volume and membrane potential as the order parameters, the self-organization models of heartbeats revealed transitions form the irregular beats to synchronized, periodic oscillations and deterministic chaos upon variation of the current of stretch-activated ion channels (control parameter). The onset of the beats frequency doubling was also analyzed using the ventricular resistance as the control parameter [141].

b) Central nervous system (CNS)

CNS is obviously one of the most complex sub-systems of the organism, revealing a multi-level architecture with strong and diverse feedback loops. The current paradigm discusses the brain as a very peculiar system, in which genuine computational features act in concert with non-computational dynamical processes, leading to continuous self-organization and remodeling under the action of external stimuli from the environment and from the rest of the organism [201]-[204]. Multiple studies demonstrated that healthy brains self-organize through activity-dependent neuroplasticity towards so-called small-world networks [205], with a combination of dense local connectivity and critical long-distance connections, revealing hierarchical modularity [206] [207]. Applying this approach, development of the brain network and its nonlinear dynamic properties were characterized with Lyapunov exponents using the sizes of the clustered nodes of the network as the order parameters [208].

The underlying idea governing neural control of behavior is the three-step structure of nervous systems, which can be described as follows: 1) Neurons transform environmental stimuli into a neural code; 2) This encoded information travels along specific pathways to the brain or central nervous system composed of nerve cells; 3) A motor instruction is generated to produce certain activity. A rigorous mathematical model of such a three-step process within the high organization level neural system is not achievable currently, but approaches describing specific functions of the neural system using bottom-up and top-down models were applied in many studies (see e.g. reviews [37] [204]).

It has been generally accepted that information processing and all functions of the brain, e.g. learning, associative learning, vision, looking and reaching, recognition, motion control, etc. are performed through complex feedback mechanisms revealing pronounced self-organized properties. Consider examples of several representa- 
tive models below.

In an example of cerebral learning, the chaotic resonance facilitated by synaptic coupling and noise was shown to enhance learning [209]. A variable that characterized the strength of phase synchronization between neurons was chosen as the order parameter, the chaotic dynamics was quantified using Lyapunov exponents, and the coupling strength between neurons was used as the control parameter.

To describe differential learning, which is regarded as a self-organized process that results in the emergence of subject- and context-dependent attractors, the generalized variable-learning rate-was chosen as the order parameter [210].

The brain's visual cortex processes information within functional (cortical) maps, which were shown to develop through self-organization process. Various studies applied different nonlinear dynamic models using specific order parameters to describe pattern formation. For example, the topological maps method allowed predicting critical segregation of the cortex into oriented columns using the generalized variables that described visual stimuli received as the order parameters [211]. In recent studies, the long-range connectivity between neurons was shown to facilitate preferential orientation maps through self-organization using generalized variables that characterized preferred orientation as the order parameter [212].

Considering the "looking and reaching" function of a vision system, recent studies suggested that only a coherent spiking activity in posterior parietal cortex could control reaches and saccades together. In addition, the neural mechanism of coordination was proposed to involve a shared collective function that acts to slow or speed movements together [213].

c) Endocrine system

The pulsatility is known to be an important inherent characteristic of hormones. Soon after the first experimental studies of the pulsatile nature of the cortisol hormone secretion by a normal man [214], it was realized that the pulsatility was not due to noise, but was associated with normal physiological processes. The circadian clock, the interaction between hormones through feedback mechanisms, and the interaction of hormones with nervous and other systems were discussed among the reasons for the pulsatile behavior. Experimental studies of hormonal systems utilizing nonlinear dynamics modelling tools allowed deeper insight into the pulsatility mechanisms [215].

The phenomenological model of three interacting hormones, namely testosterone, luteinizing hormone, and luteinizing hormone releasing hormone, was applied to describe the onset of their oscillatory behavior using hormone species densities as the order parameters [216]. The initial model of three interacting hormones was improved later in multiple works, using the rate equations model with feedback loops (see e.g. [217]).

The phase-space analysis of the hormone's dynamics was applied to investigate the pulsatile secretion in healthy humans, revealing different functioning patterns with characteristics of the low-dimensional deterministic chaos [218] and fractal attractors in the underlying dynamics of various hormones [219].

Multiple physiologically relevant models characterized bifurcations and a variety of dynamic behaviors (attractors) including oscillations, bursts, and paths to chaos, demonstrating that simple physiological feedback loops mimicking the coupling between circulatory hormones and production centers could be the source of complex hormone release patterns observed in vivo [220]-[224]. The commonly used modeling approach relied on nonlinear equations with hormone species densities as the order parameters.

\section{Applications in Medicine}

\subsection{Counterfactuals from Medicine}

The paradoxes and counterfactuals to widespread paradigms were recognized in various fields of healthcare research, indicating a need for an advanced holistic approach in studies. Recent report on evolutionary theory discussed several most unsustainable concepts of the genomic (DNA-based) evolution [225]. The analysis of health and nutrition data from various countries shows many surprising and seemingly incomprehensible facts and paradoxical relationships, e.g. the French paradox as the example of apparent contrast between "unhealthy" nutrition and low cardiovascular mortality [226]. The development of psoriatic lesions in parallel to otherwise very successful therapeutic treatment of psoriasis and psoriatic arthritis emphasized several mechanisms for the explanation of this paradoxical phenomenon, indicating a need for further system-based studies for in-depth understanding of the underlying process of disease [227]. A number of epidemiological paradoxes that have puzzled epidemiologists for a decade or more can be explained by sufficiency or lack of sun exposure hypothesis, 
pointing towards complex, system-level connections in human organisms [228]. Multiple studies discuss paradoxical drug reaction showing an outcome that is opposite from the outcome that would be expected from the drug's known actions. In bidirectional drug reactions, drugs may produce opposite effects, either in the same or different individuals, emphasizing a need for a system-level approach in pharmacokinetics and other studies [229].

One of the most pronounced examples of counterfactuals is the prevailing paradigm in cancer research that is based on somatic mutation theory with the constantly increasing catalog of genetic changes. This paradigm goes through a revision due to the growing list of inconsistencies and contradictions accumulated in cancer research [230]. A number of paradoxes of the somatic mutation theory of carcinogenesis based on the reductionist approach was indicated recently [231], calling for a more comprehensive inclusion of the advanced holistic approaches based on the concept of dynamic self-organization in cancer research.

\subsection{Oncology and Carcinogenesis}

The idea of the long-range interactions imposed by coherent dynamics, controlled by the flow of energy and information in organisms is currently seen as a generic principle of biological organization. Impairment of this flow by structural or functional, environmental or endogenous perturbations at any specific level of organization will directly destabilize the coherency and self-organizing potential of a cell or tissue. As cancer is primarily characterized in terms of progressive loss of self-organization rather than by a specific inductive agent, then impairment of the proper coherent dynamics at a specific level could be connected to such loss of self-organization. Early contributions on this topic considered the role of coherent excitations in cell division and associated implications for cancer [18] [232]. More recent studies also discussed mitochondrial dysfunction, impairment of the centrioles, disintegration of the cytoskeleton, and other relevant effects as the properties, sensitive to the biological coherency loss [233]-[235].

Carcinogenesis is one of the fields comprehensively studied with nonlinear dynamics models for multi-scale systems [236]-[238]. The normal to cancer transition is generally considered as a dynamical non-equilibrium phenomenon, which depends on both metabolic energy supply and local physiological conditions. Various nonlinear dynamics tools including entropy analysis, fractal and attractor analysis, and bifurcation analysis were applied in continuum, discrete, and hybrid models. Importantly, modeling together with in vivo clinical analysis allowed outlining preventative and therapeutic strategies.

A widely explored model describing tumor growth uses a set of coupled reaction-diffusion equations with extended network of feedbacks. Aversion of such model was applied for multiscale modeling (subcellular, cellular, diffusible, and vascular levels) of vascular tumor growth. As an example of bottom-up approach, this model accounted for coupling between layers and external stimuli. The component concentrations and expression levels were suggested as the order parameters to demonstrate the onset of the tumor growth, predict its spatiotemporal evolution, and evaluate the response to therapy [239].

The other popular approach relies on the cellular automaton (CA) discrete model that describes a spatial matrix of cells with dynamics relying on intra- and inter-cell interactions and feedbacks. In a recent example, malignant behavior of invasive solid tumors was modeled employing CA approach using the cells' density as the order parameter. The model predicted a rich spectrum of morphology, growth dynamics, and emergent behaviors of invasive tumors [240]. The authors evaluated incorporation of important environmentally controlled mechanisms in the model to apply it in clinical practice.

Physiologically relevant models of carcinogenesis suggested order parameters defined via various observable variables that identify relevant physical changes. In addition to the discussion above, the rate of cell division, cells' viscoelastic properties, mitotic index of cancer cells relative to the corresponding value for their normal counterparts, as well as changes in metabolism, $\mathrm{pH}$ values, trans-membrane potentials, spatial distribution of metabolic enzymes were proposed as possible order parameters choices for specific types of cancer [241].

\subsection{Cardiology and Electrophysiology}

Statistical and dynamical techniques applied to electrocardiogram (ECG) data clearly indicate that the frequency of the heartbeat is essentially irregular and shows patterns specific to open, far from thermodynamic equilibrium systems. The methods used to detect cardiac abnormalities in ECG recordings include stochastic processes with feedback loops [193], self-organized, turbulent electrical dynamics [242], correlation dimension method from 
chaos theory [243], multiscale entropy method for complex time series [198] [244], fractal analysis and other non-linear dynamical approaches (see e.g. review [194]).

Within the theoretical models, the transition from normal heart rate to tachycardia could be explained as corresponding to the appearance of spiral waves, and the following transition to fibrillation could be attributed to the chaotic regime after breaking up of the spiral waves [195]. From a physiological perspective, the fractal heart-rate dynamics indicates that the control mechanisms regulating the heartbeat might interact as part of a coupled cascade of feedback loops in a system operating far from equilibrium [245]. The onset of spatially discordant cardiac alternants was explained by heterogeneous nonlinear dynamics facilitated by feedback between components at different time scales and various complexity levels [136]. The physiological mechanisms underlying the dynamics and control of electrical turbulence (fibrillation) remain largely unknown; however, recent studies have demonstrated that such control is provided through the low-energy modes with efficient feedback [242].

Nonlinear dynamic models applied in cardiology and electrophysiology to describe multiscale systems used both bottom-up and top-down approaches with various (observable) macroscopic, microscopic as well as generalized variables as the order parameters. Among them were the heart rate, interbeat interval, respiratory frequency, filaments synchronization parameter, fractal dimension, Lyapunov exponents, and signal multiscale entropy.

\subsection{Clinical Neurophysiology}

Nonlinear dynamical models are used widely in neurophysiology to analyze functional magnetic resonance images (fMRI), electroencephalography (EEG) and magnetoencephalography (MEG) recordings. These models take advantage of complex interactions in neural systems, inferring processes and mechanisms at the neuronal level from measurements of brain activity and providing valuable information regarding functioning of a healthy brain as a whole system [246]. Various metrics have been applied to assess the fMRI, EEG and MEG variability, for example, using functional time-series analysis, Lyapunov exponents and multiscale entropy analysis, phasespace reconstruction techniques, bifurcation analysis, etc. [247]-[249].

Optimal brain network structural and functional organization becomes disrupted in neurological disease in characteristic ways, allowing formulation of brain disorders on the basis of qualitatively different dynamics [246]. The nonlinear dynamics tools offered unique supplemental information to the classical techniques, such as Fourier analysis, to distinguish qualitatively different fMRI/EEG/MEG recordings, e.g., in mental disorders [250] [251], in epileptic seizures [252] [253], in Parkinson's disease [246], in Alzheimer’s disease [251] [254], or in schizophrenia [251] [255]. Among the order parameters used in the models to analyze brain activity, we can indicate Kolmogorov entropy, Lyapunov exponents, fractal dimension, and signal multiscale entropy.

The analysis of brain activity using tools from nonlinear dynamics theory provide important information with regard to the underlying pharmacodynamics and therapeutic effects of different drugs, taking into consideration that the qualitative EEG/MEG changes, induced by acting drugs differ considerably (see [256] for review).

\subsection{Endocrinology}

As discussed above in Section 2.3.c, the hormonal system is multidimensional with extended feedback loops and pronounced hierarchy. Using nonlinear dynamical models to characterize the pulsatile behaviors is inevitable to properly analyze the state of the endocrine system and suggest clinical treatment of its disorders [221]. However, application of such methods in practice is currently limited to mostly qualitative, phenomenological approaches, which calls for a need for further comprehensive studies to bring appropriate nonlinear dynamic models to clinical practice. Below are a few recent examples of possible application areas.

The energy homeostasis in humans is regulated by complex mechanisms of hormonal secretion. The importance of feedback-controlled interactions with the neural system regulating appetite and satiety, reproductive function, cardiovascular system support, and other functions was pinpointed in [257]. The endocrine role of the skeleton was postulated recently based on clinical observations and analysis of the feedback loops regulating diabetes, obesity, and hypogonadism [258]. The glucocorticoids output regulation, critical for the immune, nervous, blood and other systems is not well understood and appropriate models describing important feedbacks are not available. The action of these hormones was shown to be controlled by age, gender, stress, and disease requiring construction of appropriate nonlinear models for future clinical applications [259]. One more example 
is the thyroid hormone system, which was explored extensively for many years. Recent studies indicated availability of a regulatory pathway hierarchically higher than the traditional feedback system [260]. All of these findings require further comprehensive studies with development of physiologically relevant nonlinear dynamical models.

\subsection{Other Fields of Medicine}

Besides already discussed applications in carcinogenesis, electrophysiology, endocrinology, and neurophysiology, the dynamic self-organization concept was also employed in other medical applications.

The capability of stem cells to initiate morphogenesis in vitro, generating complex structures in culture that closely parallel their in vivo counterparts was reviewed recently, discussing the mechanisms based on dynamic self-organization and evaluating applications of such models for stem cell research, disease modeling, and regenerative medicine [261]. A model studying self-organization and mineralization of vascular stent cells was used for a systems-based analysis in cardiovascular medicine [262].

The origin of complex physiologically important rhythms in living organisms, their emergence from stochastic and nonlinear biological mechanisms interacting with a fluctuating environment, as well as alterations from normal to pathological rhythm in disease states were discussed using nonlinear dynamics theories combined with physiological and medical studies [31].

An imperative role of complex mathematical models based on the dynamic self-organization concept to provide realistic insights at individual-specific optimal movement solutions in sports medicine was discussed in a number of works. Such models should incorporate a wide range of organismic, environmental and task constraints, also adopting more individualized clinical assessment procedures [263].

Complex analysis employing nonlinear dynamical methods was widely used to discuss pharmacodynamics, clinical paradoxes and bidirectional effects for drugs used in cardiovascular, CNS, dermal, endocrine, musculoskeletal, gastrointestinal, haematological, respiratory, and psychotropic agents [229] [264].

\section{Summarizing Remarks}

This review discusses a rapidly growing interest of the research community in a holistic approach to describing complex systems behavior in life sciences and medicine. The scope of the review did not allow including results of all available works evidencing or otherwise discussing importance of dynamic self-organization and coherency effects in biological systems and living organisms. We focused on providing a concise overview demonstrating the most pronounced and recent examples of such effects' existence and their importance at each level of organization in biological systems and living organisms, emphasizing the choice of the order parameters, control parameters, feedback mechanisms, and discussing importance of slaving principle in the systems' behavior.

To explore self-organization and coherency effects facilitated by feedbacks that give rise to patterns in plant and animal morphology, electrostatic waves in the heart, nonlinear dynamical effects observed in biomolecules, cells, tissues, and in other level of complexity biological entities, the entire set of available mathematical tools was used. Biological systems are inherently multilevel, composed of elements of a diverse nature in a hierarchical fashion that requires linking together different types of modeling at the various levels. At the same time, there may be no privileged level of causation in such multilevel systems with feedback loops between the scale levels [1] [5] [265]. This implies importance for a physiologically relevant model of a complex system to include relevant scales with appropriate control parameters; the analysis of the entire system might reveal at which level the order parameters might be properly defined and biological function integrated.

Successful models of dynamic self-organization in biological systems and living organisms used different tools selecting appropriate order parameters and their relations to the control parameters and system's properties. In biomolecules, the dynamic self-organization may occur due to strong charge-conformational or intra-molecular interactions causing structural changes relaxing on long time scales, exemplifying the slaving principle. This effect is facilitated by considerable flexibility of the protein environment and multiple consecutive interactions inherently characteristic to any biological system. Correspondingly, the order parameters in biomolecules are represented often through free energy functions that exemplify slow structural dynamics.

On the cell and tissue levels, the cooperative effects of multiple constituents are important contributors for the dynamic self-organization. The top level order parameters are commonly defined using the bottom-up approach 
and in most cases are represented as observable (macroscopic) variables defined through the microscopic variables or generalized variables that depend on the properties of the system's constituents.

On the organ and organism levels, available models take advantage of multiple feedbacks from the lower level entities, and the choice of the order parameters is based mainly on macroscopic variables of the system. However, using the bottom-up approach with microscopic generalized variables as order parameters also proved to be very productive in modeling organs and organisms.

In many cases of multi-scale, multi-dimensional systems, the nonlinear dynamic models used the analysis based on Lyapunov exponents and system's entropy. Such analysis allowed accurate prediction of a variety of functional modes (attractors) and regimes of switching between them, confirming the imperative role of Lyapunov exponents and system's entropy as the order parameters for modeling biological systems.

For the most complex, multiscale level systems, there are still many unknown interactions that should be specified to construct the relevant top-level models with adequate order parameters that govern the system behavior. Additional studies are required to pinpoint microscopic (low-scale) dynamics, suggesting that improved definition of order parameters through multiple local (microscopic) interactions and properties is an ongoing task for virtually all studied systems. This is especially important for the systems at the organism level and in medicine, where building comprehensive and physiologically relevant nonlinear dynamical models is still in progress.

Experimental studies of dynamic self-organization and coherent phenomena in biological systems require special attention. In many cases, experimental results targeting nonlinear dynamics effects allow rather ambiguous explanation. Requirements of examining and separation of observed properties on wide time scales, multi-modality of the observed effects, adaptive properties of biological systems, as well as the complexity and wide dynamic range of the detected signals add complexity to the analysis. However, due to recent exciting progress in introduction of powerful methods of high resolution x-ray crystallography, single molecule analysis, nuclear magnetic resonance spectroscopy, various imaging techniques, other (rather expensive) technologies as well as advances in molecular dynamics simulations, the accurate studies of nonlinear dynamics and coherency phenomena in complex biological systems acquire additional motivation.

Further studies need to tightly couple experiments with a relevant theoretical model of an inherently complex dynamic system. The experiment should target the most salient features that could not be explained outside nonlinear dynamics theories. The feedback mechanisms must be carefully analyzed to reveal the most important order parameters that determine the complex system's behavior. Experimental studies in parallel with theoretical analysis and simulations will allow deriving the quantitative measures for the order parameters and eventually making predictions about the biological system by validating the experimental data and results of modeling.

\section{Conclusions}

1) The common properties of biological systems, namely i) continuous in- and out-flow of matter and energy (open systems), ii) pronounced nonlinearities facilitated by strong feedback interactions, iii) time hierarchy for system's variables that suggest slaving principle and existence of the order parameters, and iv) dissipation that always accompanies the system's functioning support application of the dynamic self-organization concepts as defined by Prigogine [7] and Haken [9] in life sciences and healthcare research.

2) The examples provided here demonstrate that due to its far-reaching universality, the self-organization approach has the potential to afford valuable insights into homeostatic order and local interactions in complex biological systems. In this way, it can contribute to our knowledge of principles that drive the system's functions.

3) The advantage of the almost universal applicability of the dynamic self-organization theory is, however, associated with the rather ambiguous choice of specific variables - order parameters - which determine the onset of the functional processes. Therefore, it is mandatory to correlate predictions, which are derived from theoretical relations, with measured data, in order to define the physiologically relevant self-organization model for the particular biological system.

4) Among the most important order, parameters for the lowest level biological systems are those presented as generalized structural variables and expressed by the functions of specific free energies. The choice of relevant order parameters requires precise data on feedback between the system components determined at various structural levels and over a wide time scale of many decades. Computational and research tools for such analysis especially in the most complex systems are rarely available. This could be the main reason for the limitation of self-organization models proposed until recently to relatively small macromolecular, cellular and tissue species. 
The comprehensive and physiologically relevant nonlinear dynamics models for most organs as well as those related to medicine still need to be developed and/or improved.

5) Considerable progress in experimental and theoretical studies achieved during the last decades provides an additional motivation for development of realistic dynamic self-organization models in biological research and medicine. Future work will focus on the improved definition of order parameters through multiple local interactions in biological systems of various complexity levels. The impact of widely different time scales of system dynamics on order parameters will receive special attention. Another topic of interest will be a coordinated study of physiologically relevant models, which may favor a deeper understanding of the state and development of diseases and thereby provide powerful tools for healthcare research and medicine.

\section{Acknowledgments}

The authors are grateful to Prof. N.I. Kononenko and Dr. S. Viatchenko-Karpinski for critical reading of the manuscript and valuable comments. The work was partially supported by the NAS Ukraine, programs 0112-U002654 (T.O.H.) and 0112-U-000056 (L.N.C.).

\section{References}

[1] Laughlin, R. and Pines, D. (2000) The Theory of Everything. Proceedings of the National Academy of Sciences of the United States of America, 97, 28-31. http://dx.doi.org/10.1073/pnas.97.1.28

[2] Baker, S.G. and Kramer, B.S. (2011) Systems Biology and Cancer: Promises and Perils. Progress in Biophysics \& Molecular Biology, 106, 410-413. http://dx.doi.org/10.1016/j.pbiomolbio.2011.03.002

[3] Laughlin, R., Pines, D., Schmalian, J., Stojkovic, B. and Wolynes, P. (2000) The Middle Way. Proceedings of the National Academy of Sciences of the United States of America, 97, 32-37. http://dx.doi.org/10.1073/pnas.97.1.32

[4] Noble, D. (2002) Modeling the Heart—From Genes to Cells to the Whole Organ. Science, 295, 1678-1682. http://dx.doi.org/10.1126/science.1069881

[5] Qu, Z., Garfinkel, A., Weiss, J.N. and Nivala, M. (2011) Multi-Scale Modeling in Biology: How to Bridge the Gaps between Scales? Progress in Biophysics and Molecular Biology, 107, 21-31. http://dx.doi.org/10.1016/j.pbiomolbio.2011.06.004

[6] Nicolis, G. and Prigogine, I. (1977) Self-Organization in Nonequilibrium Systems: From Dissipative Structures to Order through Fluctuations. Wiley, New York.

[7] Prigogine, I. (1968) Introduction to Thermodynamics of Irreversible Processes. Interscience Publishers, New York.

[8] Haken, H. (1983) Advanced Synergetics: Instability Hierarchies of Self-Organizing Systems and Devices. SpringerVerlag, Berlin, New York.

[9] Haken, H. (1983) Synergetics: An Introduction: Nonequilibrium Phase Transitions and Self-Organization in Physics, Chemistry and Biology. Springer, Berlin.

[10] Haken, H. (1996) Slaving Principle Revisited. Physica D: Nonlinear Phenomena, 97, 95-103. http://dx.doi.org/10.1016/0167-2789(96)00080-2

[11] Benettin, G., Galgani, L. and Strelcyn, J.-M. (1976) Kolmogorov Entropy and Numerical Experiments. Physical Review A, 14, 2338-2345. http://dx.doi.org/10.1103/PhysRevA.14.2338

[12] Argoul, F. and Arneodo, A. (1986) Lyapunov Exponents and Phase Transitions in Dynamical Systems. In: Arnold, L. and Wihstutz, V., Eds., Lyapunov Exponents, Springer, Berlin, Heidelberg, 338-360.

[13] Colonna, M. and Bonasera, A. (1999) Lyapunov Exponents in Unstable Systems. Physical Review E, Statistical Physics, Plasmas, Fluids and Related Interdisciplinary Topics, 60, 444-448.

[14] Luque, B. and Solé, R.V. (2000) Lyapunov Exponents in Random Boolean Networks. Physica A: Statistical Mechanics and Its Applications, 284, 33-45. http://dx.doi.org/10.1016/S0378-4371(00)00184-9

[15] Prasad, A., Mehra, V. and Ramaswamy, R. (1997) Intermittency Route to Strange Nonchaotic Attractors. Physical Review Letters, 79, 4127-4130. http://dx.doi.org/10.1103/PhysRevLett.79.4127

[16] Schrödinger, E. (1944) What Is Life? Cambridge University Press, Cambridge.

[17] Fröhlich, H. (1968) Long-Range Coherence and Energy Storage in Biological Systems. International Journal of Quantum Chemistry, 2, 641-649.

[18] Fröhlich, H. (1978) Coherent Electric Vibrations in Biological Systems and the Cancer Problem. IEEE Transactions on Microwave Theory and Techniques, 26, 613-618. http://dx.doi.org/10.1109/TMTT.1978.1129446 
[19] Davydov, A.S. (1977) Solitons and Energy-Transfer along Protein Molecules. Journal of Theoretical Biology, 66, 377387.

[20] Cruzeiro-Hansson, L. and Takeno, S. (1997) Davydov Model: The Quantum, Mixed Quantum-Classical and Full Classical Systems. Physical Review E, 56, 894-906. http://dx.doi.org/10.1103/PhysRevE.56.894

[21] Daniel, M. and Deepamala, K. (1995) Davydov Soliton in Alpha-Helical Proteins: Higher-Order and Discreteness Effects. Physica A, 221, 241-255. http://dx.doi.org/10.1016/0378-4371(95)00243-Z

[22] Hochstrasser, D., Mertens, F. and Buttner, H. (1989) Energy-Transport by Lattice Solitons in Alpha-Helical Proteins. Physical Review A, 40, 2602-2610. http://dx.doi.org/10.1103/PhysRevA.40.2602

[23] Scott, A. (1992) Davydovs Soliton. Physics Reports, 217, 1-67. http://dx.doi.org/10.1016/0370-1573(92)90093-F

[24] Edler, J., Pfister, R., Pouthier, V., Falvo, C. and Hamm, P. (2004) Direct Observation of Self-Trapped Vibrational States in Alpha-Helices. Physical Review Letters, 93, Article ID: 106405. http://dx.doi.org/10.1103/PhysRevLett.93.106405

[25] Turing, A. (1952) The Chemical Basis of Morphogenesis. Philosophical Transactions of the Royal Society of London Series B-Biological Sciences, 237, 37-72. http://dx.doi.org/10.1098/rstb.1952.0012

[26] Kauffman, S.A. (1993) The Origins of Order: Self-Organization and Selection in Evolution. Oxford University Press, New York.

[27] Aragón, J.L., Barrio, R.A., Woolley, T.E., Baker, R.E. and Maini, P.K. (2012) Nonlinear Effects on Turing Patterns: Time Oscillations and Chaos. Physical Review E, 86, Article ID: 026201. http://dx.doi.org/10.1103/PhysRevE.86.026201

[28] Volpert, V. and Petrovskii, S. (2009) Reaction-Diffusion Waves in Biology. Physics of Life Reviews, 6, 267-310. http://dx.doi.org/10.1016/j.plrev.2009.10.002

[29] Eigen, M. (1971) Self-Organization of Matter and the Evolution of Biological Macromolecules. Naturwissenschaften, 58, 465-523.

[30] Gatenby, R.A. and Frieden, B.R. (2013) The Critical Roles of Information and Nonequilibrium Thermodynamics in Evolution of Living Systems. Bulletin of Mathematical Biology, 75, 589-601. http://dx.doi.org/10.1007/s11538-013-9821-x

[31] Glass, L. (2001) Synchronization and Rhythmic Processes in Physiology. Nature, 410, 277-284. http://dx.doi.org/10.1038/35065745

[32] Camazine, S., Ed. (2001) Self-Organization in Biological Systems. Princeton University Press, Princeton.

[33] Friston, K. (2012) A Free Energy Principle for Biological Systems. Entropy, 14, 2100-2121. http://dx.doi.org/10.3390/e14112100

[34] Karsenti, E. (2008) Self-Organization in Cell Biology: A Brief History. Nature Reviews Molecular Cell Biology, 9, 255-262. http://dx.doi.org/10.1038/nrm2357

[35] Kurakin, A. (2009) Scale-Free Flow of Life: On the Biology, Economics and Physics of the Cell. Theoretical Biology and Medical Modelling, 6, 6. http://dx.doi.org/10.1186/1742-4682-6-6

[36] Misteli, T. (2001) The Concept of Self-Organization in Cellular Architecture. The Journal of Cell Biology, 155, 181186. http://dx.doi.org/10.1083/jcb.200108110

[37] Rabinovich, M., Varona, P., Selverston, A. and Abarbanel, H. (2006) Dynamical Principles in Neuroscience. Reviews of Modern Physics, 78, 1213-1265. http://dx.doi.org/10.1103/RevModPhys.78.1213

[38] Sasai, Y. (2013) Cytosystems Dynamics in Self-Organization of Tissue Architecture. Nature, 493, 318-326. http://dx.doi.org/10.1038/nature11859

[39] Smith, N., Mulquiney, P., Nash, M., Bradley, C., Nickerson, D. and Hunter, P. (2002) Mathematical Modeling of the Heart: Cell to Organ. Chaos Solitons \& Fractals, 13, 1613-1621. http://dx.doi.org/10.1016/S0960-0779(01)00170-9

[40] Bizzarri, M., Palombo, A. and Cucina, A. (2013) Theoretical Aspects of Systems Biology. Progress in Biophysics and Molecular Biology, 112, 33-43. http://dx.doi.org/10.1016/j.pbiomolbio.2013.03.019

[41] Coffey, D. (1998) Self-Organization, Complexity and Chaos: The New Biology for Medicine. Nature Medicine, 4, 882-885. http://dx.doi.org/10.1038/nm0898-882

[42] Saetzler, K., Sonnenschein, C. and Soto, A.M. (2011) Systems Biology beyond Networks: Generating Order from Disorder through Self-Organization. Seminars in Cancer Biology, 21, 165-174. http://dx.doi.org/10.1016/j.semcancer.2011.04.004

[43] Levinthal, C. (1968) Are There Pathways for Protein Folding? Journal de Chimie Physique et de Physico-Chimie Biologique, 65, 44-45. 
[44] Fromherz, P. (1988) Self-Organization of the Fluid Mosaic of Charged Channel Proteins in Membranes. Proceedings of the National Academy of Sciences of the United States of America, 85, 6353-6357. http://dx.doi.org/10.1073/pnas.85.17.6353

[45] Binhi, V.N. and Rubin, A.B. (2007) Magnetobiology: The kT Paradox and Possible Solutions. Electromagnetic Biology and Medicine, 26, 45-62. http://dx.doi.org/10.1080/15368370701205677

[46] Bryngelson, J., Onuchic, J., Socci, N. and Wolynes, P. (1995) Funnels, Pathways and the Energy Landscape of Protein-Folding: A Synthesis. Proteins-Structure Function and Genetics, 21, 167-195. http://dx.doi.org/10.1002/prot.340210302

[47] Fersht, A. (1997) Nucleation Mechanisms in Protein Folding. Current Opinion in Structural Biology, 7, 3-9. http://dx.doi.org/10.1016/S0959-440X(97)80002-4

[48] Leopold, P., Montal, M. and Onuchic, J. (1992) Protein Folding Funnels: A Kinetic Approach to the Sequence Structure Relationship. Proceedings of the National Academy of Sciences of the United States of America, 89, 8721-8725. http://dx.doi.org/10.1073/pnas.89.18.8721

[49] Dill, K.A. and MacCallum, J.L. (2012) The Protein-Folding Problem, 50 Years On. Science, 338, 1042-1046. http://dx.doi.org/10.1126/science.1219021

[50] Wolynes, P.G. (2005) Energy Landscapes and Solved Protein-Folding Problems. Philosophical Transactions of the Royal Society A: Mathematical, Physical and Engineering Sciences, 363, 453-467. http://dx.doi.org/10.1098/rsta.2004.1502

[51] Lundgren, M., Krokhotin, A. and Niemi, A.J. (2013) Topology and Structural Self-Organization in Folded Proteins. Physical Review E, 88, Article ID: 042709. http://dx.doi.org/ 10.1103/PhysRevE.88.042709

[52] Vendruscolo, M., Zurdo, J., MacPhee, C.E. and Dobson, C.M. (2003) Protein Folding and Misfolding: A Paradigm of Self-Assembly and Regulation in Complex Biological Systems. Philosophical Transactions of the Royal Society of London Series A: Mathematical, Physical and Engineering Sciences, 361, 1205-1222. http://dx.doi.org/10.1098/rsta.2003.1194

[53] Gerstman, B. and Chapagain, P. (2005) Self-Organization in Protein Folding and the Hydrophobic Interaction. Journal of Chemical Physics, 123, Article ID: 054901. http://dx.doi.org/10.1063/1.1990110

[54] Malinovska, L., Kroschwald, S. and Alberti, S. (2013) Protein Disorder, Prion Propensities and Self-Organizing Macromolecular Collectives. Biochimica et Biophysica Acta (BBA)_Proteins and Proteomics, 1834, 918-931. http://dx.doi.org/10.1016/j.bbapap.2013.01.003

[55] Nelson, E. and Onuchic, J. (1998) Proposed Mechanism for Stability of Proteins to Evolutionary Mutations. Proceedings of the National Academy of Sciences of the United States of America, 95, 10682-10686. http://dx.doi.org/10.1073/pnas.95.18.10682

[56] Batey, S., Randles, L., Steward, A. and Clarke, J. (2005) Cooperative Folding in a Multi-Domain Protein. Journal of Molecular Biology, 349, 1045-1059. http://dx.doi.org/10.1016/j.jmb.2005.04.028

[57] Finkelstein, A.V. and Galzitskaya, O.V. (2004) Physics of Protein Folding. Physics of Life Reviews, 1, 23-56. http://dx.doi.org/10.1016/j.plrev.2004.03.001

[58] Kuznetsova, I., Turoverov, K. and Uversky, V. (2004) Use of the Phase Diagram Method to Analyze the Protein Unfolding-Refolding Reactions: Fishing out the "Invisible” Intermediates. Journal of Proteome Research, 3, 485-494. http://dx.doi.org/10.1021/pr034094y

[59] Lin, M.M. and Zewail, A.H. (2012) Protein Folding—Simplicity in Complexity. Annalen Der Physik, 524, $379-391$. http://dx.doi.org/10.1002/andp.201200501

[60] Vendruscolo, M. and Dobson, C.M. (2005) Towards Complete Descriptions of the Free-Energy Landscapes of Proteins. Philosophical Transactions of the Royal Society A: Mathematical, Physical and Engineering Sciences, 363, 433-452. http://dx.doi.org/10.1098/rsta.2004.1501

[61] Wong, G.C.L. and Pollack, L. (2010) Electrostatics of Strongly Charged Biological Polymers: Ion-Mediated Interactions and Self-Organization in Nucleic Acids and Proteins. Annual Review of Physical Chemistry, 61, 171-189. http://dx.doi.org/10.1146/annurev.physchem.58.032806.104436

[62] Komar, A.A. (2009) A Pause for Thought along the Co-Translational Folding Pathway. Trends in Biochemical Sciences, 34, 16-24. http://dx.doi.org/10.1016/j.tibs.2008.10.002

[63] Pechmann, S. and Frydman, J. (2013) Evolutionary Conservation of Codon Optimality Reveals Hidden Signatures of Cotranslational Folding. Nature Structural \& Molecular Biology, 20, 237-243. http://dx.doi.org/10.1038/nsmb.2466

[64] Simister, P.C., Schaper, F., O’Reilly, N., McGowan, S. and Feller, S.M. (2011) Self-Organization and Regulation of Intrinsically Disordered Proteins with Folded N-Termini. Plos Biology, 9, e1000591. http://dx.doi.org/10.1371/journal.pbio.1000591 
[65] Astumian, R.D. (1997) Thermodynamics and Kinetics of a Brownian Motor. Science, 276, 917-922. http://dx.doi.org/10.1126/science.276.5314.917

[66] Jülicher, F., Ajdari, A. and Prost, J. (1997) Modeling Molecular Motors. Reviews of Modern Physics, 69, $1269-1282$. http://dx.doi.org/10.1103/RevModPhys.69.1269

[67] Surrey, T., Nedelec, F., Leibler, S. and Karsenti, E. (2001) Physical Properties Determining Self-Organization of Motors and Microtubules. Science, 292, 1167-1171. http://dx.doi.org/10.1126/science.1059758

[68] Geislinger, B., Darnell, E., Farris, K. and Kawai, R. (2005) Are Motor Proteins Power Strokers, Brownian Motors or Both? (Invited Paper). In: Kish, L.B., Lindenberg, K. and Gingl, Z., Eds., Proceedings of SPIE 5845, Noise in Complex Systems and Stochastic Dynamics III, 93-103. http://dx.doi.org/10.1117/12.609464

[69] Ge, H. and Qian, H. (2013) Dissipation, Generalized Free Energy and a Self-Consistent Nonequilibrium Thermodynamics of Chemically Driven Open Subsystems. Physical Review E, 87, Article ID: 062125. http://dx.doi.org/10.1103/PhysRevE.87.062125

[70] Howard, J. (2009) Mechanical Signaling in Networks of Motor and Cytoskeletal Proteins. Annual Review of Biophysics, 38, 217-234. http://dx.doi.org/10.1146/annurev.biophys.050708.133732

[71] Vogel, S.K., Pavin, N., Maghelli, N., Jülicher, F. and Tolić-Nørrelykke, I.M. (2010) Microtubules and Motor Proteins: Mechanically Regulated Self-Organization in Vivo. The European Physical Journal Special Topics, 178, 57-69. http://dx.doi.org/10.1140/epjst/e2010-01182-6

[72] Hodgkin, A. and Huxley, A. (1952) A Quantitative Description of Membrane Current and Its Application to Conduction and Excitation in Nerve. Journal of Physiology-London, 117, 500-544.

[73] Hodgkin, A. (1964) The Ionic Basis of Nervous Conduction. Science, 145, 1148-1154. http://dx.doi.org/10.1126/science.145.3637.1148

[74] Hilt, M. and Zimmermann, W. (2007) Hexagonal, Square and Stripe Patterns of the Ion Channel Density in Biomembranes. Physical Review E, 75, Article ID: 016202. http://dx.doi.org/10.1103/PhysRevE.75.016202

[75] Leonetti, M., Nuebler, J. and Homble, F. (2006) Parity-Breaking Bifurcation and Global Oscillation in Patterns of Ion Channels. Physical Review Letters, 96, Article ID: 218101. http://dx.doi.org/10.1103/PhysRevLett.96.218101

[76] Jensen, M.O., Jogini, V., Borhani, D.W., Leffler, A.E., Dror, R.O. and Shaw, D.E. (2012) Mechanism of Voltage Gating in Potassium Channels. Science, 336, 229-233. http://dx.doi.org/10.1126/science.1216533

[77] Chinarov, V., Gaididei, Y., Kharkyanen, V. and Sitko, S. (1992) Ion Pores in Biological-Membranes as Self-Organized Bistable Systems. Physical Review A, 46, 5232-5241. http://dx.doi.org/10.1103/PhysRevA.46.5232

[78] Amaral, C., Carnevale, V., Klein, M.L. and Treptow, W. (2012) Exploring Conformational States of the Bacterial Voltage-Gated Sodium Channel NavAb via Molecular Dynamics Simulations. Proceedings of the National Academy of Sciences of the United States of America, 109, 21336-21341. http://dx.doi.org/10.1073/pnas.1218087109

[79] Bjelkmar, P., Niemelä, P.S., Vattulainen, I. and Lindahl, E. (2009) Conformational Changes and Slow Dynamics through Microsecond Polarized Atomistic Molecular Simulation of an Integral Kv1.2 Ion Channel. PLoS Computational Biology, 5, e1000289. http://dx.doi.org/10.1371/journal.pcbi.1000289

[80] Haider, S., Grottesi, A., Hall, B.A., Ashcroft, F.M. and Sansom, M.S.P. (2005) Conformational Dynamics of the Ligand-Binding Domain of Inward Rectifier K Channels as Revealed by Molecular Dynamics Simulations: Toward an Understanding of Kir Channel Gating. Biophysical Journal, 88, 3310-3320. http://dx.doi.org/10.1529/biophysj.104.052019

[81] McCusker, E.C., Bagneris, C., Naylor, C.E., Cole, A.R., D’Avanzo, N., Nichols, C.G., et al. (2012) Structure of a Bacterial Voltage-Gated Sodium Channel Pore Reveals Mechanisms of Opening and Closing. Nature Communications, $\mathbf{3}$, Article Number: 1102. http://dx.doi.org/10.1038/ncomms2077

[82] Payandeh, J., Scheuer, T., Zheng, N. and Catterall, W.A. (2011) The Crystal Structure of a Voltage-Gated Sodium Channel. Nature, 475, 353-358. http://dx.doi.org/10.1038/nature10238

[83] Tayefeh, S., Kloss, T., Kreim, M., Gebhardt, M., Baumeister, D., Hertel, B., et al. (2009) Model Development for the Viral Kcv Potassium Channel. Biophysical Journal, 96, 485-498. http://dx.doi.org/10.1016/j.bpj.2008.09.050

[84] Vargas, E., Yarov-Yarovoy, V., Khalili-Araghi, F., Catterall, W.A., Klein, M.L., Tarek, M., et al. (2012) An Emerging Consensus on Voltage-Dependent Gating from Computational Modeling and Molecular Dynamics Simulations. The Journal of General Physiology, 140, 587-594. http://dx.doi.org/10.1085/jgp.201210873

[85] Christophorov, L.N., Kharkyanen, V.N. and Sit'ko, S.P. (1991) On the Concept of the Non-Equilibrium Conformon (Self-Organization of a Selected Degree of Freedom in Biomolecular Systems). Journal of Biological Physics, 18, 191202.

[86] Zarubin, D., Zhuchkova, E. and Schreiber, S. (2012) Effects of Cooperative Ion-Channel Interactions on the Dynamics of Excitable Membranes. Physical Review E, 85, Article ID: 061904. http://dx.doi.org/10.1103/PhysRevE.85.061904 
[87] Park, C., Shcheglovitov, A. and Dolmetsch, R. (2010) The CRAC Channel Activator STIM1 Binds and Inhibits LType Voltage-Gated Calcium Channels. Science, 330, 101-105. http://dx.doi.org/10.1126/science.1191027

[88] Jung, P., Swaminathan, D. and Ullah, A. (2010) Calcium Spikes: Chance or Necessity? Chemical Physics, 375, 625629. http://dx.doi.org/10.1016/j.chemphys.2010.05.010

[89] Katona, G., Snijder, A., Gourdon, P., Andréasson, U., Hansson, Ö., Andréasson, L.-E., et al. (2005) Conformational Regulation of Charge Recombination Reactions in a Photosynthetic Bacterial Reaction Center. Nature Structural \& Molecular Biology, 12, 630-631. http://dx.doi.org/10.1038/nsmb948

[90] Kleinfeld, D., Okamura, M. and Feher, G. (1984) Electron-Transfer Kinetics in Photosynthetic Reaction Centers Cooled to Cryogenic Temperatures in the Charge-Separated State-Evidence for Light-Induced Structural-Changes. Biochemistry, 23, 5780-5786. http://dx.doi.org/10.1021/bi00319a017

[91] Kononenko, A., Noks, P., Chamorovskii, S., Rubin, A., Likhtenshtein, G., Krupyanskii, Y., et al. (1986) ElectronTransfer and Intermolecular Dynamics of Photosynthetic Reaction Centers. Khimicheskaya Fizika, 5, 795-804.

[92] Wohri, A.B., Katona, G., Johansson, L.C., Fritz, E., Malmerberg, E., Andersson, M., et al. (2010) Light-Induced Structural Changes in a Photosynthetic Reaction Center Caught by Laue Diffraction. Science, 328, 630-633. http://dx.doi.org/10.1126/science.1186159

[93] Christophorov, L.N. (1995) Conformation-Dependent Charge-Transport-A New Stochastic Approach. Physics Letters A, 205, 14-17. http://dx.doi.org/10.1016/0375-9601(95)00462-C

[94] Goushcha, A.O., Kharkyanen, V., Scott, G. and Holzwarth, A. (2000) Self-Regulation Phenomena in Bacterial Reaction Centers. I. General Theory. Biophysical Journal, 79, 1237-1252.

[95] Andréasson, U. and Andréasson, L.-E. (2003) Characterization of a Semi-Stable, Charge-Separated State in Reaction Centers from Rhodobacter sphaeroides. Photosynthesis Research, 75, 223-233. http://dx.doi.org/10.1023/A:1023944605460

[96] Deshmukh, S.S., Williams, J.C., Allen, J.P. and Kálmán, L. (2011) Light-Induced Conformational Changes in Photosynthetic Reaction Centers: Dielectric Relaxation in the Vicinity of the Dimer. Biochemistry, 50, 340-348. http://dx.doi.org/10.1021/bi101496c

[97] Malferrari, M., Mezzetti, A., Francia, F. and Venturoli, G. (2013) Effects of Dehydration on Light-Induced Conformational Changes in Bacterial Photosynthetic Reaction Centers Probed by Optical and Differential FTIR Spectroscopy. Biochimica et Biophysica Acta (BBA)—Bioenergetics, 1827, 328-339. http://dx.doi.org/10.1016/j.bbabio.2012.10.009

[98] Christophorov, L.N. and Kharkyanen, V.N. (2005) Synergetic Mechanisms of Structural Regulation of the Electron Transfer and Other Reactions of Biological Macromolecules. Chemical Physics, 319, 330-341. http://dx.doi.org/10.1016/j.chemphys.2005.06.029

[99] Christophorov, L.N., Kharkyanen, V.N. and Berezetskaya, N.M. (2013) Molecular Self-Organization: A Single Molecule Aspect. Chemical Physics Letters, 583, 170-174. http://dx.doi.org/10.1016/j.cplett.2013.08.005

[100] Tributsch, H. and Pohlmann, L. (1997) Synergetic Electron Transfer in Molecular Electronic and Photosynthetic Mechanisms. Journal of Electroanalytical Chemistry, 438, 37-41. http://dx.doi.org/10.1016/S0022-0728(96)05061-9

[101] Kirchhoff, H., Haase, W., Haferkamp, S., Schott, T., Borinski, M., Kubitscheck, U., et al. (2007) Structural and Functional Self-Organization of Photosystem II in Grana Thylakoids. Biochimica et Biophysica Acta (BBA)—Bioenergetics, 1767, 1180-1188. http://dx.doi.org/10.1016/j.bbabio.2007.05.009

[102] Engel, G.S., Calhoun, T.R., Read, E.L., Ahn, T.-K., Mancal, T., Cheng, Y.-C., et al. (2007) Evidence for Wavelike Energy Transfer through Quantum Coherence in Photosynthetic Systems. Nature, 446, 782-786. http://dx.doi.org/10.1038/nature05678

[103] Novoderezhkin, V., Monshouwer, R. and van Grondelle, R. (2000) Electronic and Vibrational Coherence in the Core Light-Harvesting Antenna of Rhodopseudomonas viridis. Journal of Physical Chemistry B, 104, 12056-12071. http://dx.doi.org/10.1021/jp001881z

[104] Prokhorenko, V., Holzwarth, A., Nowak, F. and Aartsma, T. (2002) Growing-In of Optical Coherence in the FMO Antenna Complexes. Journal of Physical Chemistry B, 106, 9923-9933. http://dx.doi.org/10.1021/jp025758e

[105] Collini, E., Wong, C.Y., Wilk, K.E., Curmi, P.M.G., Brumer, P. and Scholes, G.D. (2010) Coherently Wired LightHarvesting in Photosynthetic Marine Algae at Ambient Temperature. Nature, 463, 644-647. http://dx.doi.org/10.1038/nature08811

[106] Hildner, R., Brinks, D., Nieder, J.B., Cogdell, R.J. and van Hulst, N.F. (2013) Quantum Coherent Energy Transfer over Varying Pathways in Single Light-Harvesting Complexes. Science, 340, 1448-1451. http://dx.doi.org/10.1126/science.1235820

[107] Levitz, J., Pantoja, C., Gaub, B., Janovjak, H., Reiner, A., Hoagland, A., et al. (2013) Optical Control of Metabotropic Glutamate Receptors. Nature Neuroscience, 16, 507-516. http://dx.doi.org/10.1038/nn.3346 
[108] Vasiliauskas, D., Mazzoni, E.O., Sprecher, S.G., Brodetskiy, K., Johnston Jr., R.J., Lidder, P., et al. (2011) Feedback from Rhodopsin Controls Rhodopsin Exclusion in Drosophila Photoreceptors. Nature, 479, 108-112. http://dx.doi.org/10.1038/nature10451

[109] Field, G.D. and Rieke, F. (2002) Mechanisms Regulating Variability of the Single Photon Responses of Mammalian Rod Photoreceptors. Neuron, 35, 733-747. http://dx.doi.org/10.1016/S0896-6273(02)00822-X

[110] Whitlock, G. and Lamb, T. (1999) Variability in the Time Course of Single Photon Responses from Toad Rods Termination of Rhodopsin's Activity. Neuron, 23, 337-351. http://dx.doi.org/10.1016/S0896-6273(00)80784-9

[111] Caruso, G., Bisegna, P., Andreucci, D., Lenoci, L., Gurevich, V.V., Hamm, H.E., et al. (2011) Identification of Key Factors That Reduce the Variability of the Single Photon Response. Proceedings of the National Academy of Sciences of the United States of America, 108, 7804-7807. http://dx.doi.org/10.1073/pnas.1018960108

[112] Pumir, A., Graves, J., Ranganathan, R. and Shraiman, B.I. (2008) Systems Analysis of the Single Photon Response in Invertebrate Photoreceptors. Proceedings of the National Academy of Sciences of the United States of America, 105, 10354-10359. http://dx.doi.org/10.1073/pnas.0711884105

[113] Smith, E., Krishnamurthy, S., Fontana, W. and Krakauer, D. (2011) Nonequilibrium Phase Transitions in Biomolecular Signal Transduction. Physical Review E, 84, Article ID: 051917. http://dx.doi.org/10.1103/PhysRevE.84.051917

[114] Prokhorenko, V., Nagy, A.M., Waschuk, S.A., Brown, L.S., Birge, R.R. and Miller, R.J.D. (2006) Coherent Control of Retinal Isomerization in Bacteriorhodopsin. Science, 313, 1257-1261. http://dx.doi.org/10.1126/science.1130747

[115] Kraack, J.P., Buckup, T. and Motzkus, M. (2013) Coherent High-Frequency Vibrational Dynamics in the Excited Electronic State of All-Trans Retinal Derivatives. Journal of Physical Chemistry Letters, 4, 383-387. http://dx.doi.org/10.1021/jz302001m

[116] Weingart, O. and Garavelli, M. (2012) Modelling Vibrational Coherence in the Primary Rhodopsin Photoproduct. Journal of Chemical Physics, 137, Article ID: 22A523. http://dx.doi.org/10.1063/1.4742814

[117] Liebl, U., Lipowski, G., Negrerie, M., Lambry, J., Martin, J. and Vos, M. (1999) Coherent Reaction Dynamics in a Bacterial Cytochrome C Oxidase. Nature, 401, 181-184.

[118] Varga, V., Leduc, C., Bormuth, V., Diez, S. and Howard, J. (2009) Kinesin-8 Motors Act Cooperatively to Mediate Length-Dependent Microtubule Depolymerization. Cell, 138, 1174-1183. http://dx.doi.org/10.1016/j.cell.2009.07.032

[119] Julicher, F., Kruse, K., Prost, J. and Joanny, J. (2007) Active Behavior of the Cytoskeleton. Physics Reports, 449, 3-28. http://dx.doi.org/10.1016/j.physrep.2007.02.018

[120] Sumino, Y., Nagai, K.H., Shitaka, Y., Tanaka, D., Yoshikawa, K., Chate, H., et al. (2012) Large-Scale Vortex Lattice Emerging from Collectively Moving Microtubules. Nature, 483, 448-452. http://dx.doi.org/10.1038/nature10874

[121] Hussain, S., Molloy, J.E. and Khan, S.M. (2013) Spatiotemporal Dynamics of Actomyosin Networks. Biophysical Journal, 105, 1456-1465. http://dx.doi.org/10.1016/j.bpj.2013.08.001

[122] Bornens, M. (1989) The Cortical Microfilament System of Lymphoblasts Displays a Periodic Oscillatory Activity in the Absence of Microtubules: Implications for Cell Polarity. The Journal of Cell Biology, 109, 1071-1083. http://dx.doi.org/10.1083/jcb.109.3.1071

[123] Pelling, A.E. (2004) Local Nanomechanical Motion of the Cell Wall of Saccharomyces cerevisiae. Science, 305, 11471150. http://dx.doi.org/10.1126/science. 1097640

[124] Levayer, R. and Lecuit, T. (2012) Biomechanical Regulation of Contractility: Spatial Control and Dynamics. Trends in Cell Biology, 22, 61-81. http://dx.doi.org/10.1016/j.tcb.2011.10.001

[125] Bendix, P.M., Koenderink, G.H., Cuvelier, D., Dogic, Z., Koeleman, B.N., Brieher, W.M., et al. (2008) A Quantitative Analysis of Contractility in Active Cytoskeletal Protein Networks. Biophysical Journal, 94, 3126-3136. http://dx.doi.org/10.1529/biophysj.107.117960

[126] Koehler, S., Schaller, V. and Bausch, A.R. (2011) Collective Dynamics of Active Cytoskeletal Networks. PLoS ONE, 6, e23798. http://dx.doi.org/10.1371/journal.pone.0023798

[127] Wang, S. and Wolynes, P. (2012) Active Contractility in Actomyosin Networks. Proceedings of the National Academy of Sciences of the United States of America, 109, 6446-6451. http://dx.doi.org/10.1073/pnas.1204205109

[128] Salbreux, G., Joanny, J.F., Prost, J. and Pullarkat, P. (2007) Shape Oscillations of Non-Adhering Fibroblast Cells. Physical Biology, 4, 268-284. http://dx.doi.org/10.1088/1478-3975/4/4/004

[129] Chay, T. and Lee, Y. (1985) Phase Resetting and Bifurcation in the Ventricular Myocardium. Biophysical Journal, 47, 641-651.

[130] Jung, P. and Gailey, P.C. (2000) The Heartbeat of Extended Clocks. Annalen der Physik, 9, 697-704. http://dx.doi.org/10.1002/1521-3889(200010)9:9/10<697::AID-ANDP697>3.0.CO;2-B

[131] Qu, Z., Nivala, M. and Weiss, J.N. (2013) Calcium Alternans in Cardiac Myocytes: Order from Disorder. Journal of 
Molecular and Cellular Cardiology, 58, 100-109. http://dx.doi.org/10.1016/j.yjmcc.2012.10.007

[132] Cheng, H., Lederer, M., Lederer, W. and Cannell, M. (1996) Calcium Sparks and [Ca2+]i Waves in Cardiac Myocytes. American Journal of Physiology-Cell Physiology, 270, C148-C159.

[133] Lechleiter, J., Girard, S., Peralta, E. and Clapham, D. (1991) Spiral Calcium Wave-Propagation and Annihilation in Xenopus-Laevis Oocytes. Science, 252, 123-126. http://dx.doi.org/10.1126/science.2011747

[134] Lipp, P. and Niggli, E. (1993) Microscopic Spiral Waves Reveal Positive Feedback in Subcellular Calcium Signaling. Biophysical Journal, 65, 2272-2276.

[135] Marchant, J. and Parker, I. (2001) Role of Elementary $\mathrm{Ca}^{2+}$ Puffs in Generating Repetitive $\mathrm{Ca}^{2+}$ Oscillations. EMBO Journal, 20, 65-76. http://dx.doi.org/10.1093/emboj/20.1.65

[136] Weiss, J.N. (2006) From Pulsus to Pulseless: The Saga of Cardiac Alternans. Circulation Research, 98, $1244-1253$. http://dx.doi.org/10.1161/01.RES.0000224540.97431.f0

[137] Skardal, P.S., Karma, A. and Restrepo, J.G. (2012) Unidirectional Pinning and Hysteresis of Spatially Discordant Alternans in Cardiac Tissue. Physical Review Letters, 108, Article ID: 108103. http://dx.doi.org/10.1103/PhysRevLett.108.108103

[138] Tran, D., Sato, D., Yochelis, A., Weiss, J., Garfinkel, A. and Qu, Z. (2009) Bifurcation and Chaos in a Model of Cardiac Early after Depolarizations. Physical Review Letters, 102, Article ID: 258103. http://dx.doi.org/10.1103/PhysRevLett.102.258103

[139] Grosu, R., Smolka, S.A., Corradini, F., Wasilewska, A., Entcheva, E. and Bartocci, E. (2009) Learning and Detecting Emergent Behavior in Networks of Cardiac Myocytes. Communications of the ACM, 52, 97-105. http://dx.doi.org/10.1145/1467247.1467271

[140] Restrepo, J. and Karma, A. (2009) Line-Defect Patterns of Unstable Spiral Waves in Cardiac Tissue. Physical Review E, 79, Article ID: 030906. http://dx.doi.org/10.1103/PhysRevE.79.030906

[141] Hori, S., Yamaguchi, Y. and Shimizu, H. (1999) Self-Organization of the Heartbeat as Coordination among Ventricular Myocardial Cells through Mechano-Electrical Feedback. Biological Cybernetics, 80, 1-10. http://dx.doi.org/10.1007/s004220050500

[142] Asby, W.R. (1960) Design for a Brain. John Wiley and Sons, Inc., New York, 286 p.

[143] Block, H. (1962) The Perceptron: A Model for Brain Functioning. I. Reviews of Modern Physics, 34, 123-135. http://dx.doi.org/10.1103/RevModPhys.34.123

[144] Rosenblatt, F. (1962) Principles of Neurodynamics: Perceptions and the Theory of Brain Mechanisms. Spartan Books, New York.

[145] Willshaw, D. and Malsburg, C. (1976) How Patterned Neural Connections Can Be Set up by Self-Organization. Proceedings of the Royal Society Series B-Biological Sciences, 194, 431-445. http://dx.doi.org/10.1098/rspb.1976.0087

[146] Aihara, K., Numajiri, T., Matsumoto, G. and Kotani, M. (1986) Structures of Attractors in Periodically Forced Neural Oscillators. Physics Letters A, 116, 313-317. http://dx.doi.org/10.1016/0375-9601(86)90578-5

[147] Canavier, C., Baxter, D., Clark, J. and Byrne, J. (1993) Nonlinear Dynamics in a Model Neuron Provide a Novel Mechanism for Transient Synaptic Inputs to Produce Long-Term Alterations of Postsynaptic Activity. Journal of Neurophysiology, 69, 2252-2257.

[148] Chay, T. (1984) Abnormal Discharges and Chaos in a Neuronal Model System. Biological Cybernetics, 50, $301-311$. http://dx.doi.org/10.1007/BF00337079

[149] Ermentrout, B. (2010) Mathematical Foundations of Neuroscience. Springer, New York.

[150] Ibarz, B., Casado, J.M. and Sanjuan, M.A.F. (2011) Map-Based Models in Neuronal Dynamics. Physics Reports-Review Section of Physics Letters, 501, 1-74. http://dx.doi.org/10.1016/j.physrep.2010.12.003

[151] Rothman, J.S., Cathala, L., Steuber, V. and Silver, R.A. (2009) Synaptic Depression Enables Neuronal Gain Control. Nature, 457, 1015-1018. http://dx.doi.org/10.1038/nature07604

[152] Guckenheimer, J., Gueron, S. and Harriswarrick, R. (1993) Mapping the Dynamics of a Bursting Neuron. Philosophical Transactions of the Royal Society of London Series B-Biological Sciences, 341, 345-359. http://dx.doi.org/10.1098/rstb.1993.0121

[153] Komendantov, A.O. and Kononenko, N.I. (1996) Deterministic Chaos in Mathematical Model of Pacemaker Activity in Bursting Neurons of Snail, Helix Pomatia. Journal of Theoretical Biology, 183, 219-230. http://dx.doi.org/10.1006/jtbi.1996.0215

[154] Li, Y., Schmid, G., Hänggi, P. and Schimansky-Geier, L. (2010) Spontaneous Spiking in an Autaptic Hodgkin-Huxley Setup. Physical Review E, 82, Article ID: 061907. http://dx.doi.org/10.1103/PhysRevE.82.061907

[155] Hirata, Y., Oku, M. and Aihara, K. (2012) Chaos in Neurons and Its Application: Perspective of Chaos Engineering. 
Chaos, 22, Article ID: 047511. http://dx.doi.org/10.1063/1.4738191

[156] Yuste, R. and Denk, W. (1995) Dendritic Spines as Basic Functional Units of Neuronal Integration. Nature, 375, 682684. http://dx.doi.org/10.1038/375682a0

[157] Legenstein, R. and Maass, W. (2011) Branch-Specific Plasticity Enables Self-Organization of Nonlinear Computation in Single Neurons. Journal of Neuroscience, 31, 10787-10802. http://dx.doi.org/10.1523/JNEUROSCI.5684-10.2011

[158] Ahmed, W.W., Williams, B.J., Silver, A.M. and Saif, T.A. (2013) Measuring Nonequilibrium Vesicle Dynamics in Neurons under Tension. Lab on a Chip, 13, 570-578. http://dx.doi.org/10.1039/c2lc41109a

[159] Hebb, D.O. (1949) The Organization of Behavior. A Neuropsychological Theory. John Wiley and Sons, Inc., New York.

[160] Fuhrmann, G., Segev, I., Markram, H. and Tsodyks, M. (2002) Coding of Temporal Information by Activity-Dependent Synapses. Journal of Neurophysiology, 87, 140-148.

[161] Hennig, M.H. (2013) Theoretical Models of Synaptic Short Term Plasticity. Frontiers in Computational Neuroscience, 7, Article Number: 45. http://dx.doi.org/10.3389/fncom.2013.00045

[162] O’Donnell, C., Nolan, M.F. and van Rossum, M.C.W. (2011) Dendritic Spine Dynamics Regulate the Long-Term Stability of Synaptic Plasticity. Journal of Neuroscience, 31, 16142-16156. http://dx.doi.org/10.1523/JNEUROSCI.2520-11.2011

[163] Saneyoshi, T., Fortin, D.A. and Soderling, T.R. (2010) Regulation of Spine and Synapse Formation by Activity-Dependent Intracellular Signaling Pathways. Current Opinion in Neurobiology, 20, 108-115. http://dx.doi.org/10.1016/j.conb.2009.09.013

[164] Abbott, L.F. and Nelson, S.B. (2000) Synaptic Plasticity: Taming the Beast. Nature Neuroscience, 3, 1178-1183. http://dx.doi.org/10.1038/81453

[165] Colon-Ramos, D.A. (2009) Synapse Formation in Developing Neural Circuits. In: Hobert, O., Ed., Development of Neural Circuitry, Elsvevier Academic Press Inc., San Diego, 53-79.

[166] Bi, G. and Poo, M. (1998) Synaptic Modifications in Cultured Hippocampal Neurons: Dependence on Spike Timing, Synaptic Strength and Postsynaptic Cell Type. Journal of Neuroscience, 18, 10464-10472.

[167] Caporale, N. and Dan, Y. (2008) Spike Timing-Dependent Plasticity: A Hebbian Learning Rule. Annual Review of Neuroscience, 31, 25-46. http://dx.doi.org/10.1146/annurev.neuro.31.060407.125639

[168] Clopath, C., Buesing, L., Vasilaki, E. and Gerstner, W. (2010) Connectivity Reflects Coding: A Model of VoltageBased STDP with Homeostasis. Nature Neuroscience, 13, 344-352. http://dx.doi.org/10.1038/nn.2479

[169] Cox, K.J.A. and Adams, P.R. (2009) Hebbian Crosstalk Prevents Nonlinear Unsupervised Learning. Frontiers in Computational Neuroscience, 3, 11. http://dx.doi.org/10.3389/neuro.10.011.2009

[170] Elliott, T. (2012) Cross-Talk Induces Bifurcations in Nonlinear Models of Synaptic Plasticity. Neural Computation, 24, 455-522. http://dx.doi.org/10.1162/NECO_a_00224

[171] Popovych, O.V., Yanchuk, S. and Tass, P.A. (2013) Self-Organized Noise Resistance of Oscillatory Neural Networks with Spike Timing-Dependent Plasticity. Scientific Reports, 3, Article Number: 2926. http://dx.doi.org/10.1038/srep02926

[172] Golding, N., Staff, N. and Spruston, N. (2002) Dendritic Spikes as a Mechanism for Cooperative Long-Term Potentiation. Nature, 418, 326-331. http://dx.doi.org/10.1038/nature00854

[173] Harnett, M.T., Makara, J.K., Spruston, N., Kath, W.L. and Magee, J.C. (2012) Synaptic Amplification by Dendritic Spines Enhances Input Cooperativity. Nature, 491, 599-602. http://dx.doi.org/10.1038/nature11554

[174] Sjostrom, P.J. and Hausser, M. (2006) A Cooperative Switch Determines the Sign of Synaptic Plasticity in Distal Dendrites of Neocortical Pyramidal Neurons. Neuron, 51, 227-238. http://dx.doi.org/10.1016/j.neuron.2006.06.017

[175] Buzsáki, G. (2010) Neural Syntax: Cell Assemblies, Synapsembles and Readers. Neuron, 68, 362-385. http://dx.doi.org/10.1016/j.neuron.2010.09.023

[176] Dupont, E., Hanganu, I.L., Kilb, W., Hirsch, S. and Luhmann, H.J. (2005) Rapid Developmental Switch in the Mechanisms Driving Early Cortical Columnar Networks. Nature, 439, 79-83. http://dx.doi.org/10.1038/nature04264

[177] Hopfield, J. (1982) Neural Networks and Physical Systems with Emergent Collective Computational Abilities. Proceedings of the National Academy of Sciences of the United States of America, 79, 2554-2558. http://dx.doi.org/10.1073/pnas.79.8.2554

[178] Strogatz, S.H. (2001) Exploring Complex Networks. Nature, 410, 268-276. http://dx.doi.org/10.1038/35065725

[179] Van Vreeswijk, C. and Sompolinsky, H. (1996) Chaos in Neuronal Networks with Balanced Excitatory and Inhibitory Activity. Science, 274, 1724-1726. 
[180] Huang, X. (2004) Spiral Waves in Disinhibited Mammalian Neocortex. The Journal of Neuroscience, 24, $9897-9902$. http://dx.doi.org/10.1523/JNEUROSCI.2705-04.2004

[181] Pinto, D.J. and Ermentrout, G.B. (2001) Spatially Structured Activity in Synaptically Coupled Neuronal Networks: I. Traveling Fronts and Pulses. SIAM Journal on Applied Mathematics, 62, 206-225. http://dx.doi.org/10.1137/S0036139900346453

[182] Wilson, H.R. and Cowan, J.D. (1972) Excitatory and Inhibitory Interactions in Localized Populations of Model Neurons. Biophysical Journal, 12, 1-24. http://dx.doi.org/10.1016/S0006-3495(72)86068-5

[183] Lazar, A. (2009) SORN: A Self-Organizing Recurrent Neural Network. Frontiers in Computational Neuroscience, 3, 23. http://dx.doi.org/10.3389/neuro.10.023.2009

[184] Zheng, P., Dimitrakakis, C. and Triesch, J. (2013) Network Self-Organization Explains the Statistics and Dynamics of Synaptic Connection Strengths in Cortex. Plos Computational Biology, 9, e1002848. http://dx.doi.org/10.1371/journal.pcbi.1002848

[185] Fries, P. (2005) A Mechanism for Cognitive Dynamics: Neuronal Communication through Neuronal Coherence. Trends in Cognitive Sciences, 9, 474-480. http://dx.doi.org/10.1016/j.tics.2005.08.011

[186] Akam, T., Oren, I., Mantoan, L., Ferenczi, E. and Kullmann, D.M. (2012) Oscillatory Dynamics in the Hippocampus Support Dentate Gyrus-CA3 Coupling. Nature Neuroscience, 15, 763-768. http://dx.doi.org/10.1038/nn.3081

[187] Bressloff, P.C. and Newby, J.M. (2013) Stochastic Models of Intracellular Transport. Reviews of Modern Physics, 85, 135-196. http://dx.doi.org/10.1103/RevModPhys.85.135

[188] Junkin, M., Leung, S.L., Whitman, S., Gregorio, C.C. and Wong, P.K. (2011) Cellular Self-Organization by Autocatalytic Alignment Feedback. Journal of Cell Science, 124, 4213-4220. http://dx.doi.org/10.1242/jcs.088898

[189] Qian, H. (2012) Cooperativity in Cellular Biochemical Processes: Noise-Enhanced Sensitivity, Fluctuating Enzyme, Bistability with Nonlinear Feedback and Other Mechanisms for Sigmoidal Responses. Annual Review of Biophysics, 41, 179-204. http://dx.doi.org/10.1146/annurev-biophys-050511-102240

[190] Tyson, J., Chen, K. and Novak, B. (2003) Sniffers, Buzzers, Toggles and Blinkers: Dynamics of Regulatory and Signaling Pathways in the Cell. Current Opinion in Cell Biology, 15, 221-231. http://dx.doi.org/10.1016/S0955-0674(03)00017-6

[191] Bray, D. (2009) Wetware: A Computer in Every Living Cell. Yale University Press, New Haven, London.

[192] Santos, S.D.M., Verveer, P.J. and Bastiaens, P.I.H. (2007) Growth Factor-Induced MAPK Network Topology Shapes Erk Response Determining PC-12 Cell Fate. Nature Cell Biology, 9, 324-330. http://dx.doi.org/10.1038/ncb1543

[193] Ivanov, P., Amaral, L., Goldberger, A. and Stanley, H. (1998) Stochastic Feedback and the Regulation of Biological Rhythms. Europhysics Letters, 43, 363-368. http://dx.doi.org/10.1209/epl/i1998-00366-3

[194] Shiogai, Y., Stefanovska, A. and McClintock, P.V.E. (2010) Nonlinear Dynamics of Cardiovascular Ageing. Physics Reports, 488, 51-110. http://dx.doi.org/10.1016/j.physrep.2009.12.003

[195] Wagner, C. and Persson, P. (1998) Chaos in the Cardiovascular System: An Update. Cardiovascular Research, 40, 257-264. http://dx.doi.org/10.1016/S0008-6363(98)00251-X

[196] Moorman, J.R., Delos, J.B., Flower, A.A., Cao, H., Kovatchev, B.P., Richman, J.S., et al. (2011) Cardiovascular Oscillations at the Bedside: Early Diagnosis of Neonatal Sepsis Using Heart Rate Characteristics Monitoring. Physiological Measurement, 32, 1821-1832. http://dx.doi.org/10.1088/0967-3334/32/11/S08

[197] Aubert, A.E., Vandeput, S., Beckers, F., Liu, J., Verheyden, B. and Van Huffel, S. (2009) Complexity of Cardiovascular Regulation in Small Animals. Philosophical Transactions of the Royal Society A: Mathematical, Physical and Engineering Sciences, 367, 1239-1250. http://dx.doi.org/10.1098/rsta.2008.0276

[198] Costa, M.D., Peng, C.-K. and Goldberger, A.L. (2008) Multiscale Analysis of Heart Rate Dynamics: Entropy and Time Irreversibility Measures. Cardiovascular Engineering, 8, 88-93. http://dx.doi.org/10.1007/s10558-007-9049-1

[199] Perkiömäki, J., Mäkikallio, T. and Huikuri, H. (2005) Fractal and Complexity Measures of Heart Rate Variability. Clinical and Experimental Hypertension, 27, 149-158. http://dx.doi.org/10.1081/CEH-200048742

[200] Kohl, P. and Sachs, F. (2001) Mechanoelectric Feedback in Cardiac Cells. Philosophical Transactions of the Royal Society A-Mathematical Physical and Engineering Sciences, 359, 1173-1185. http://dx.doi.org/10.1098/rsta.2001.0824

[201] Garrett, D.D., Samanez-Larkin, G.R., MacDonald, S.W.S., Lindenberger, U., McIntosh, A.R. and Grady, C.L. (2013) Moment-to-Moment Brain Signal Variability: A Next Frontier in Human Brain Mapping? Neuroscience and Biobehavioral Reviews, 37, 610-624. http://dx.doi.org/10.1016/j.neubiorev.2013.02.015

[202] Guidolin, D., Albertin, G., Guescini, M., Fuxe, K. and Agnati, L.F. (2011) Central Nervous System and Computation. The Quarterly Review of Biology, 86, 265-285. http://dx.doi.org/10.1086/662456

[203] Haken, H. (2007) Brain Dynamics: Synchronization and Activity Patterns in Pulse-Coupled Neural Nets with Delays 
and Noise. Springer, Berlin, New York.

[204] Schoner, G. and Kelso, J. (1988) Dynamic Pattern Generation in Behavioral and Neural Systems. Science, 239, 15131520. http://dx.doi.org/10.1126/science.3281253

[205] Watts, D. and Strogatz, S. (1998) Collective Dynamics of “Small-World” Networks. Nature, 393, 440-442. http://dx.doi.org/10.1038/30918

[206] Park, H. and Friston, K. (2013) Structural and Functional Brain Networks: From Connections to Cognition. Science, 342, 1238411-1238411. http://dx.doi.org/10.1126/science.1238411

[207] Stam, C.J. and van Straaten, E.C.W. (2012) The Organization of Physiological Brain Networks. Clinical Neurophysiology, 123, 1067-1087. http://dx.doi.org/10.1016/j.clinph.2012.01.011

[208] Rubinov, M., Sporns, O., van Leeuwen, C. and Breakspear, M. (2009) Symbiotic Relationship between Brain Structure and Dynamics. BMC Neuroscience, 10, 55. http://dx.doi.org/10.1186/1471-2202-10-55

[209] Tokuda, I.T., Han, C.E., Aihara, K., Kawato, M. and Schweighofer, N. (2010) The Role of Chaotic Resonance in Cerebellar Learning. Neural Networks, 23, 836-842. http://dx.doi.org/10.1016/j.neunet.2010.04.006

[210] Frank, T.D., Michelbrink, M., Beckmann, H. and Schöllhorn, W.I. (2007) A Quantitative Dynamical Systems Approach to Differential Learning: Self-Organization Principle and Order Parameter Equations. Biological Cybernetics, 98, 19-31. http://dx.doi.org/10.1007/s00422-007-0193-x

[211] Obermayer, K., Blasdel, G. and Schulten, K. (1992) Statistical-Mechanical Analysis of Self-Organization and Pattern Formation during the Development of Visual Maps. Physical Review A, 45, 7568-7589. http://dx.doi.org/10.1103/PhysRevA.45.7568

[212] Kaschube, M., Schnabel, M., Lowel, S., Coppola, D.M., White, L.E. and Wolf, F. (2010) Universality in the Evolution of Orientation Columns in the Visual Cortex. Science, 330, 1113-1116. http://dx.doi.org/10.1126/science.1194869

[213] Dean, H.L., Hagan, M.A. and Pesaran, B. (2012) Only Coherent Spiking in Posterior Parietal Cortex Coordinates Looking and Reaching. Neuron, 73, 829-841. http://dx.doi.org/10.1016/j.neuron.2011.12.035

[214] Hellman, L., Nakada, F., Curti, J., Weitzman, E., Kream, J., Roffwarg, H., et al. (1970) Cortisol Is Secreted Episodically by Normal Man. Journal of Clinical Endocrinology \& Metabolism, 30, 411-422.

[215] Veldhuis, J., Keenan, D.M. and Pincus, S.M. (2008) Motivations and Methods for Analyzing Pulsatile Hormone Secretion. Endocrine Reviews, 29, 823-864. http://dx.doi.org/10.1210/er.2008-0005

[216] Smith, W. (1980) Hypothalamic Regulation of Pituitary Secretion of Luteinizing Hormone-II Feedback Control of Gonadotropin Secretion. Bulletin of Mathematical Biology, 42, 57-78. http://dx.doi.org/10.1007/BF02462366

[217] Greenhalgh, D. and Khan, Q.J.A. (2009) A Delay Differential Equation Mathematical Model for the Control of the Hormonal System of the Hypothalamus, the Pituitary and the Testis in Man. Nonlinear Analysis: Theory, Methods \& Applications, 71, e925-e935. http://dx.doi.org/10.1016/j.na.2009.01.031

[218] Prank, K., Harms, H., Dammig, M., Brabant, G., Mitschke, F. and Hesch, R. (1994) Is There Low-Dimensional Chaos in Pulsatile Secretion of Parathyroid-Hormone in Normal Human-Subjects. American Journal of Physiology, 266, E653-E658.

[219] Papavasiliou, S.S., Brue, T., Jaquet, P. and Castanas, E. (1995) Pattern of Prolactin Diurnal Secretion in Normal Humans: Evidence for Nonlinear Dynamics. Neuroendocrinology, 62, 444-453. http://dx.doi.org/10.1159/000127034

[220] Hamann, H., Schmickl, T. and Crailsheim, K. (2012) A Hormone-Based Controller for Evaluation-Minimal Evolution in Decentrally Controlled Systems. Artificial Life, 18, 165-198.

[221] Keenan, D.M., Wang, X., Pincus, S.M. and Veldhuis, J.D. (2012) Modeling the Nonlinear Time Dynamics of Multidimensional Hormonal Systems: Time Dynamics of Hormonal Systems. Journal of Time Series Analysis, 33, 779-796. http://dx.doi.org/10.1111/j.1467-9892.2012.00795.x

[222] Londergan, C. and Peacock-Lopez, E. (1998) Dynamic Model of Hormonal Systems Coupled by Negative Feedback. Biophysical Chemistry, 73, 85-107. http://dx.doi.org/10.1016/S0301-4622(98)00140-9

[223] Sriram, K., Rodriguez-Fernandez, M. and Doyle, F.J. (2012) Modeling Cortisol Dynamics in the Neuro-Endocrine Axis Distinguishes Normal, Depression and Post-Traumatic Stress Disorder (PTSD) in Humans. PLoS Computational Biology, 8, e1002379. http://dx.doi.org/10.1371/journal.pcbi.1002379

[224] Zhusubaliyev, Z.T., Churilov, A.N. and Medvedev, A. (2012) Bifurcation Phenomena in an Impulsive Model of NonBasal Testosterone Regulation. Chaos, 22, Article ID: 013121. http://dx.doi.org/10.1063/1.3685519

[225] Shapiro, J.A. (2013) Rethinking the (Im) Possible in Evolution. Progress in Biophysics \& Molecular Biology, 111, 9296. http://dx.doi.org/10.1016/j.pbiomolbio.2012.08.016

[226] Ginter, E., Simko, V. and Dolinska, S. (2009) Paradoxes in Medicine: An Access to New Knowledge? Bratislava Medical Journal-Bratislavske Lekarske Listy, 110, 112-115. 
[227] Aslanidis, S., Pyrpasopoulou, A., Douma, S. and Triantafyllou, A. (2008) Tumor Necrosis Factor-A Antagonist-Induced Psoriasis: Yet Another Paradox in Medicine. Clinical Rheumatology, 27, 377-380. http://dx.doi.org/10.1007/s10067-007-0789-5

[228] Gillie, O. (2012) The Scots’ Paradox: Can Sun Exposure, or Lack of it, Explain Major Paradoxes in Epidemiology? Anticancer Research, 32, 237-248.

[229] Smith, S., Hauben, M. and Aronson, J.K. (2012) Paradoxical and Bidirectional Drug Effects. Drug Safety, 35, $173-189$.

[230] Baker, S.G., Cappuccio, A. and Potter, J.D. (2010) Research on Early-Stage Carcinogenesis: Are We Approaching Paradigm Instability? Journal of Clinical Oncology, 28, 3215-3218. http://dx.doi.org/10.1200/JCO.2010.28.5460

[231] Baker, S.G. and Kramer, B.S. (2007) Paradoxes in Carcinogenesis: New Opportunities for Research Directions. BMC Cancer, 7, 151. http://dx.doi.org/10.1186/1471-2407-7-151

[232] Hyland, G.J. (2009) Frohlich’s Coherent Excitations \& the Cancer Problem-A Retrospecive Overview of His Guiding Philosophy1. Electromagnetic Biology and Medicine, 28, 316-329. http://dx.doi.org/10.1080/15368370802708827

[233] Ao, P., Galas, D., Hood, L. and Zhu, X. (2008) Cancer as Robust Intrinsic State of Endogenous Molecular-Cellular Network Shaped by Evolution. Medical Hypotheses, 70, 678-684. http://dx.doi.org/10.1016/j.mehy.2007.03.043

[234] Plankar, M., Del Giudice, E., Tedeschi, A. and Jerman, I. (2012) The Role of Coherence in a Systems View of Cancer Development. Theoretical Biology Forum, 105, 15-46.

[235] Pokorny, J. (2009) Biophysical Cancer Transformation Pathway. Electromagnetic Biology and Medicine, 28, 105-123. http://dx.doi.org/10.1080/15368370802711615

[236] Deisboeck, T.S. and Couzin, I.D. (2009) Collective Behavior in Cancer Cell Populations. BioEssays, 31, 190-197. http://dx.doi.org/10.1002/bies.200800084

[237] Dinicola, S., D’Anselmi, F., Pasqualato, A., Proietti, S., Lisi, E., Cucina, A., et al. (2011) A Systems Biology Approach to Cancer: Fractals, Attractors and Nonlinear Dynamics. OMICS: A Journal of Integrative Biology, 15, 93-104. http://dx.doi.org/10.1089/omi.2010.0091

[238] Khain, E. and Sander, L. (2006) Dynamics and Pattern Formation in Invasive Tumor Growth. Physical Review Letters, 96, Article ID: 188103. http://dx.doi.org/10.1103/PhysRevLett.96.188103

[239] Perfahl, H., Byrne, H.M., Chen, T., Estrella, V., Alarcón, T., Lapin, A., et al. (2011) Multiscale Modeling of Vascular Tumour Growth in 3D: The Roles of Domain Size and Boundary Conditions. PLoS ONE, 6, e14790. http://dx.doi.org/10.1371/journal.pone.0014790

[240] Jiao, Y. and Torquato, S. (2013) Evolution and Morphology of Microenvironment-Enhanced Malignancy of Three-Dimensional Invasive Solid Tumors. Physical Review E, 87, Article ID: 052707. http://dx.doi.org/10.1103/PhysRevE.87.052707

[241] Davies, P.C.W., Demetrius, L. and Tuszynski, J.A. (2011) Cancer as a Dynamical Phase Transition. Theoretical Biology and Medical Modeling, 8, 30. http://dx.doi.org/10.1186/1742-4682-8-30

[242] Luther, S., Fenton, F.H., Kornreich, B.G., Squires, A., Bittihn, P., Hornung, D., et al. (2011) Low-Energy Control of Electrical Turbulence in the Heart. Nature, 475, 235-239. http://dx.doi.org/10.1038/nature10216

[243] Bogaert, C., Beckers, F., Ramaekers, D. and Aubert, A. (2001) Analysis of Heart Rate Variability with Correlation Dimension Method in a Normal Population and in Heart Transplant Patients. Autonomic Neuroscience-Basic \& Clinical, 90, 142-147. http://dx.doi.org/10.1016/S1566-0702(01)00280-6

[244] Richman, J. and Moorman, J. (2000) Physiological Time-Series Analysis Using Approximate Entropy and Sample Entropy. American Journal of Physiology-Heart and Circulatory Physiology, 278, H2039-H2049.

[245] Ivanov, P., Amaral, L., Goldberger, A., Havlin, S., Rosenblum, M., Struzik, Z., et al. (1999) Multifractality in Human Heartbeat Dynamics. Nature, 399, 461-465. http://dx.doi.org/10.1038/20924

[246] Stam, C. (2005) Nonlinear Dynamical Analysis of EEG and MEG: Review of an Emerging Field. Clinical Neurophysiology, 116, 2266-2301. http://dx.doi.org/10.1016/j.clinph.2005.06.011

[247] El Boustani, S. and Destexhe, A. (2010) Brain Dynamics at Multiple Scales: Can One Reconcile the Apparent Low-Dimensional Chaos of Macroscopic Variables with the Seemingly Stochastic Behavior of Single Neurons? International Journal of Bifurcation and Chaos, 20, 1687-1702. http://dx.doi.org/10.1142/S0218127410026769

[248] Phothisonothai, M. and Nakagawa, M. (2007) Fractal-Based EEG Data Analysis of Body Parts Movement Imagery Tasks. Journal of Physiological Sciences, 57, 217-226. http://dx.doi.org/10.2170/physiolsci.RP006307

[249] Stephan, K.E., Kasper, L., Harrison, L.M., Daunizeau, J., den Ouden, H.E.M., Breakspear, M., et al. (2008) Nonlinear Dynamic Causal Models for fMRI. NeuroImage, 42, 649-662. http://dx.doi.org/10.1016/j.neuroimage.2008.04.262

[250] Bosl, W., Tierney, A., Tager-Flusberg, H. and Nelson, C. (2011) EEG Complexity as a Biomarker for Autism Spectrum Disorder Risk. BMC Medicine, 9, 18. http://dx.doi.org/10.1186/1741-7015-9-18 
[251] Takahashi, T. (2013) Complexity of Spontaneous Brain Activity in Mental Disorders. Progress in Neuro-Psychopharmacology \& Biological Psychiatry, 45, 258-266. http://dx.doi.org/10.1016/j.pnpbp.2012.05.001

[252] Lehnertz, K. (2008) Epilepsy and Nonlinear Dynamics. Journal of Biological Physics, 34, 253-266. http://dx.doi.org/10.1007/s10867-008-9090-3

[253] Silva, C., Pimentel, I., Andrade, A., Foreid, J. and Ducla-Soares, E. (1999) Correlation Dimension Maps of EEG from Epileptic Absences. Brain Topography, 11, 201-209. http://dx.doi.org/10.1023/A:1022281712161

[254] Arendt, T. (2005) Alzheimer’s Disease as a Disorder of Dynamic Brain Self-Organization. Progress in Brain Research, 147, 355-378.

[255] Carlino, E., Sigaudo, M., Pollo, A., Benedetti, F., Mongini, T., Castagna, F., et al. (2012) Nonlinear Analysis of Electroencephalogram at Rest and during Cognitive Tasks in Patients with Schizophrenia. Journal of Psychiatry \& Neuroscience, 37, 259-266. http://dx.doi.org/10.1503/jpn.110030

[256] Bewernitz, M. and Derendorf, H. (2012) Electroencephalogram-Based Pharmacodynamic Measures: A Review. International Journal of Clinical Pharmacology and Therapeutics, 50, 162-184. http://dx.doi.org/10.5414/CP201484

[257] Fuqua, J.S. and Rogol, A.D. (2013) Neuroendocrine Alterations in the Exercising Human: Implications for Energy Homeostasis. Metabolism, 62, 911-921. http://dx.doi.org/10.1016/j.metabol.2013.01.016

[258] Schwetz, V., Pieber, T. and Obermayer-Pietsch, B. (2012) Mechanisms in Endocrinology: The Endocrine Role of the Skeleton: Background and Clinical Evidence. European Journal of Endocrinology, 166, 959-967. http://dx.doi.org/10.1530/EJE-12-0030

[259] Veldhuis, J., Sharma, A. and Roelfsema, F. (2013) Age-Dependent and Gender-Dependent Regulation of Hypothalamic-Adrenocorticotropic-Adrenal Axis. Endocrinology and Metabolism Clinics of North America, 42, 201-225. http://dx.doi.org/10.1016/j.ecl.2013.02.002

[260] Costa-e-Sousa, R.H. and Hollenberg, A.N. (2012) Minireview: The Neural Regulation of the Hypothalamic-PituitaryThyroid Axis. Endocrinology, 153, 4128-4135. http://dx.doi.org/10.1210/en.2012-1467

[261] Sato, T. and Clevers, H. (2013) Growing Self-Organizing Mini-Guts from a Single Intestinal Stem Cell: Mechanism and Applications. Science, 340, 1190-1194. http://dx.doi.org/10.1126/science.1234852

[262] Sage, A., Tintut, Y., Garfinkel, A. and Demer, L. (2009) Systems Biology of Vascular Calcification. Trends in Cardiovascular Medicine, 19, 118-123.

[263] Glazier, P.S. and Davids, K. (2009) Constraints on the Complete Optimization of Human Motion. Sports Medicine, 39, 15-28.

[264] Dokoumetzidis, A., Iliadis, A. and Macheras, P. (2001) Nonlinear Dynamics and Chaos Theory: Concepts and Applications Relevant to Pharmacodynamics. Pharmaceutical Research, 18, 415-426. http://dx.doi.org/10.1023/A:1011083723190

[265] Noble, D. (2011) A Theory of Biological Relativity: No Privileged Level of Causation. Interface Focus, 2, 55-64. http://dx.doi.org/10.1098/rsfs.2011.0067 
Scientific Research Publishing (SCIRP) is one of the largest Open Access journal publishers. It is currently publishing more than 200 open access, online, peer-reviewed journals covering a wide range of academic disciplines. SCIRP serves the worldwide academic communities and contributes to the progress and application of science with its publication.

Other selected journals from SCIRP are listed as below. Submit your manuscript to us via either submit@scirp.org or Online Submission Portal.
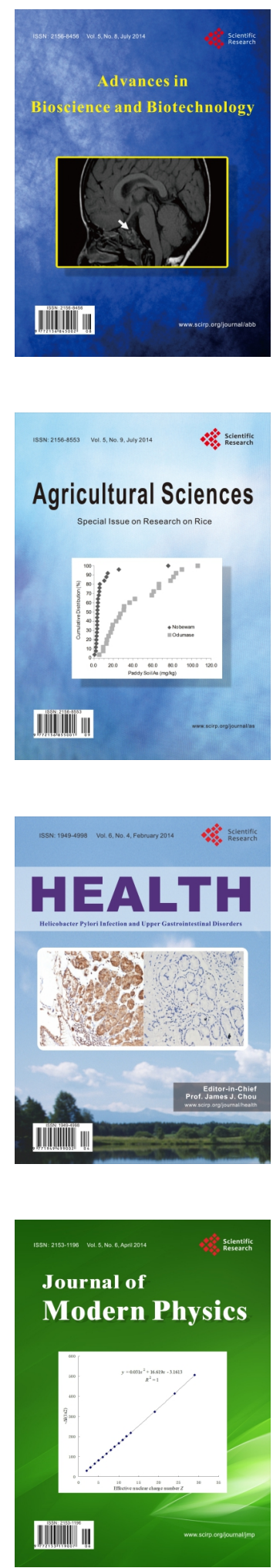
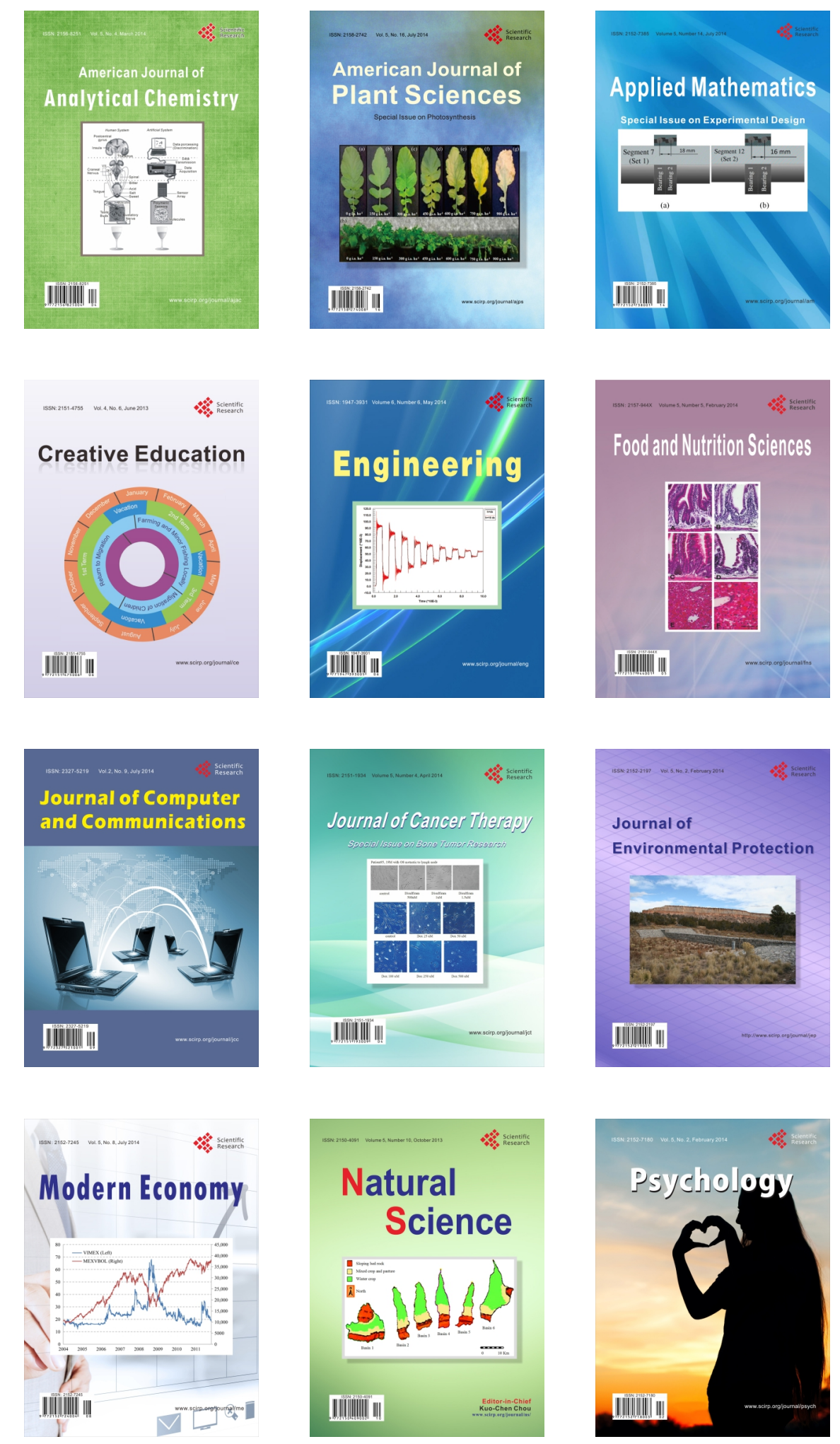